\title{
STICKELBERGER SERIES AND MAIN CONJECTURE FOR FUNCTION FIELDS
}

\author{
ANDREA BANDINI AND EDOARDO COSCELLI
}

\begin{abstract}
Let $F$ be a global function field of characteristic $p$ with ring of integers $A$ and let $\Phi$ be a Hayes module on the Hilbert class field $H_{A}$ of $F$. We prove an Iwasawa Main Conjecture for the $\mathbb{Z}_{p}^{\infty}$-extension $\mathcal{F} / F$ generated by the $\mathfrak{p}$-power torsion of $\Phi$ (p a prime of $A$ ). The main tool is a Stickelberger series whose specialization provides a generator for the Fitting ideal of the class group of $\mathcal{F}$. Moreover we prove that the same series, evaluated at complex or p-adic characters, interpolates the Goss Zeta-function or some p-adic $L$-function, thus providing the link between the algebraic structure (class groups) and the analytic functions, which is the crucial part of Iwasawa Main Conjecture.
\end{abstract}

\section{INTRODUCTION}

Arithmetic properties of motives defined over a global field are a central theme of modern number theory and one of the main topic is their relation with (or interpretation as) special values of $L$-functions. Iwasawa theory combines an algebraic approach, which studies the variation of motives in $p$-adic towers as modules over an algebra of power series, with the definition of analytical $p$-adic $L$-functions, which interpolate special values of more classical $L$-functions, thus providing a link between the two aspects of the theory. The Main Conjecture (IMC) predicts (in a growing number of incarnations) a deep relation between $p$-adic $L$-functions and a generator of the characteristic or Fitting ideal of the algebraic structure we deal with (class groups, Selmer groups and so on).

This topic has been extensively studied since the first proof of a Main Conjecture by Mazur and Wiles (in [22]) for the class groups of the $\mathbb{Z}_{p}$-cyclotomic extension of $\mathbb{Q}$, but mostly for $\mathbb{Z}_{p}$ or $\mathbb{Z}_{p}^{d}$-extensions of number fields (see e.g. [20]). Iwasawa theory for function fields in positive characteristic is relatively new and, even if some instances of the IMC for $\mathbb{Z}_{p}^{d}$ extensions were proved by Crew in [13] and Burns, Lai and Tan in [9], we believe that the true analog of the Mazur-Wiles theorem is the one recently presented in [2]. We briefly explain the setting of that result: let $F=\mathbb{F}_{q}(t)$ be the rational function field which plays the role of $\mathbb{Q}$, fix the prime $\frac{1}{t}=\infty$ and consider $A=\mathbb{F}_{q}[t]$ as the ring of integers of $F$, i.e. the functions regular outside $\infty$. Let $\mathfrak{p}$ be a prime of $A$ and let $F_{n}$ be the field generated over $F$ by the $\mathfrak{p}^{n+1}$-torsion of the Carlitz module associated to $A$ (see [2, Section 2] for a quick review): put $\mathcal{F}=\cup F_{n}$, then $\operatorname{Gal}(\mathcal{F} / F) \simeq \operatorname{Gal}\left(F_{0} / F\right) \times \operatorname{Gal}\left(\mathcal{F} / F_{0}\right) \simeq \Delta \times \mathbb{Z}_{p}^{\infty}$, where $\Delta$ has finite order prime with $p$. The field $\mathcal{F}$ is the analog of the cyclotomic $\mathbb{Z}_{p}$-extension of a number field constructed with $p$-power roots of unity and it is this analogy which led us to believe that we have to work with $\mathcal{F}$ instead of $\mathbb{Z}_{p}^{d}$-extensions for some finite $d$.

2010 Mathematics Subject Classification. 11R23, 11R60, 11R58, 11M38, 11S40 .

Key words and phrases. Function fields, Main Conjecture, p-adic $L$-functions, Stickelberger series, divisor class groups. 
This provides one of the main differences with the number field setting where there are no $\mathbb{Z}_{p}^{\infty}$-extensions: here our algebraic structures (namely class groups) will be modules over the non-noetherian algebra $\Lambda(\mathcal{F})=W \llbracket \operatorname{Gal}\left(\mathcal{F} / F_{0}\right) \rrbracket(W$ an appropriate finite extension of $\mathbb{Z}_{p}$ ) and, having no structure theorem for them, we shall use a limit process to describe their Fitting ideals.

Let $\mathcal{C} \ell_{n}^{0}$ be the $p$-part of the group of divisor classes of degree 0 of $F_{n}$ and, for any complex character $\chi$ of $\Delta$, let $\mathcal{C} \ell_{n}^{0}(\chi)$ be its $\chi$-part, i.e. the one on which $\Delta$ acts via $\chi$. Put $\mathcal{C} \ell_{\infty}^{0}(\chi)$ for the inverse limit with respect to norms of the $\mathcal{C} \ell_{n}^{0}(\chi)$, then [2, Theorems 5.1 and 5.2] prove that for any nontrivial $\chi$ the $\Lambda(\mathcal{F})$-module $\mathcal{C} \ell_{\infty}^{0}(\chi)$ is finitely generated and torsion and its Fitting ideal is generated by a specialization of the $\chi$-part of a Stickelberger series $\Theta(X) \in \mathbb{Z}[\operatorname{Gal}(\mathcal{F} / F)] \llbracket X \rrbracket$.

The same Stickelberger series is also used to interpolate (via complex or $\mathfrak{p}$-adic characters) the Artin $L$-function, the Goss Zeta-function and a p-adic $L$-function ([2, Section 3]), thus providing the link between the algebraic structure of $\mathcal{C} \ell_{\infty}^{0}(\chi)$ and various $L$-functions, i.e. the IMC.

1.1. Our results. Our goal here is to extend the main results of [2] to a general function field $F$ of characteristic $p$, i.e. a field of transcendence degree 1 over some finite field $\mathbb{F}_{q}$ or, equivalently, the function field of a smooth projective curve defined over $\mathbb{F}_{q}$. Fix a prime $\infty$, let $A$ be the ring of integers for $F$ and write $H_{A}$ for the Hilbert class field of $A$, i.e. the maximal abelian unramified extension of $F$ where $\infty$ is totally split.

To deal with this setting we need to consider extensions generated by the $\mathfrak{p}$-torsion of a signnormalized rank 1 Drinfeld module (or Hayes module, see Definition 5.2 and decompose Iwasawa modules in eigenspaces with respect to characters whose action on the inertia groups of $\infty$ is crucial. We replace the odd and even characters of the case $F=\mathbb{F}_{q}(t)$ (where $H_{A}=F$ ) with three types (Definition 5.8): we shall provide a complete study for the first two types, the characters of type 3 need a different treatment (see Remark 1.1). Moreover the presence of a nontrivial Hilbert class field $H_{A}$ enters in the definition of the Goss Zeta-function (in particular in the exponentiation of ideals) and we have to take it into account in all our interpolation formulas and in the definition of our $\mathfrak{p}$-adic $L$-series. Fix a finite set $S$ of primes of $F$ and let $F_{S}$ be the maximal abelian extension of $F$ unramified outside $S$ with $G_{S}:=\operatorname{Gal}\left(F_{S} / F\right)$. For any prime $\nu$, let $d_{\nu}$ be its degree and, if $\nu \notin S$, denote by $\phi_{\nu}$ the Frobenius of $\nu$ in $G_{S}$. The leading role in this paper will be played by the Stickelberger series

$$
\Theta_{S}(X)=\prod_{\nu \notin S}\left(1-\phi_{\nu}^{-1} X^{d_{\nu}}\right)^{-1} \in \mathbb{Z}\left[G_{S}\right] \llbracket X \rrbracket .
$$

1.1.1. Analytic side. In Sections 2 , 3 and 4 we prove convergence properties for $\Theta_{S}(X)$ and test it against characters $\Psi: \mathbb{Z}\left[G_{S}\right] \longrightarrow L$ to provide interpolation formulas for

- the Artin $L$-function $L_{F}$ (for $L=\mathbb{C}$, Theorem 2.3);

- the Goss Zeta-function $\zeta_{A}$ (for $L=\mathbb{C}_{\infty}$, Theorem 3.8);

- various $\nu$-adic Zeta-functions $\zeta_{\nu}$ (for $L=\mathbb{C}_{\nu}$, Theorem 4.4),

where $\mathbb{C}_{\eta}$ is the completion of an algebraic closure of the completion $F_{\eta}$ of $F$ at a prime $\eta$ (for both cases $\eta$ finite or infinite). Then we construct a $\nu$-adic $L$-series which, via its relation with $\Theta_{S}(X)$, interpolates the values of $\zeta_{\nu}$ at integers for $\nu \in S-\{\infty\}$ (and a bit more, see Theorem 4.9 . 
As an example we mention here the interpolation we obtain for the Goss Zeta-function

$$
\zeta_{A}(s)=\sum \mathfrak{a}^{-s} \quad \text { with } s \in \mathbb{S}_{\infty}:=\mathbb{C}_{\infty}^{\times} \times \mathbb{Z}_{p},
$$

where the sum is taken over all the nonzero ideals $\mathfrak{a}$ of $A$. This function represents the natural analogue of the Dedekind Zeta-function of a number field. For any $y \in \mathbb{Z}_{p}$ we define a continuous character $\Psi_{y}: G_{S} \rightarrow \mathbb{C}_{\infty}^{\times}$via

$$
\Psi_{y}\left(\phi_{\nu}\right)=N\left(\langle\nu\rangle_{\infty}^{-1}\right)^{y / f}
$$

(details in Section 3.2, here it suffices to say that $N$ is a norm, $f$ is a power of $p$ and $\langle\nu\rangle_{\infty}$ is a 1 -unit so that taking the $\frac{y}{f}$-th power makes sense). For every $s=(x, y) \in \mathbb{S}_{\infty}$ we have

$$
\Psi_{y}\left(\Theta_{S}(X)\right)(x)=\zeta_{A}(-s) \prod_{\nu \in S, \nu \neq \infty}\left(1-\nu^{s}\right) .
$$

1.1.2. Algebraic side. Let $\Phi: A \rightarrow H_{A}\{\tau\}$ be a Hayes module (more details on all objects mentioned here are in Section 5p: fix a prime $\mathfrak{p}$ of $F$ with degree $d_{\mathfrak{p}}$ and denote by $F_{n}$ the extension of $H_{A}$ generated by the $\mathfrak{p}^{n+1}$-torsion of $\Phi$. The field $F_{n}$ is an abelian Galois extension of $F$ ramified only at $\mathfrak{p}$ and $\infty$. These fields form an Iwasawa tower

$$
F \subset H_{A} \subset F_{0} \subset F_{1} \subset \cdots \subset F_{n} \subset \cdots \subset \bigcup_{n \in \mathbb{N}} F_{n}=: \mathcal{F}
$$

and, if we put $\Gamma_{n}=\operatorname{Gal}\left(F_{n} / F_{0}\right)$, we have

$$
\Gamma_{\infty}:=\operatorname{Gal}\left(\mathcal{F} / F_{0}\right)=\lim _{\leftarrow} \operatorname{Gal}\left(F_{n} / F_{0}\right) \simeq \mathbb{Z}_{p}^{\infty} .
$$

Let $C_{n}:=\mathcal{C} \ell^{0}\left(F_{n}\right)$ be the $p$-part of the class group of degree zero divisors of $F_{n}$ : it is a $\mathbb{Z}_{p}\left[\Gamma_{n}\right]$-module in a natural way and the $C_{n}$ form a projective system with respect to the norm maps. We define $C_{\infty}:=\lim _{\leftarrow} C_{n}$, which is a module over the non-noetherian Iwasawa algebra $\mathbb{Z}_{p} \llbracket \Gamma_{\infty} \rrbracket$. To study the structure of $C_{\infty}$ we consider $\chi$-parts with respect to the characters of the group $G_{0}:=\operatorname{Gal}\left(F_{0} / F\right)$ which acts naturally on $C_{n}$ and $C_{\infty}$. We need two simplifying assumptions (which are not required for the analytic part of the theory):

- $\operatorname{deg}(\infty)=d_{\infty}=1$, this ensures that all extensions we work with are geometric but it is not restrictive because we can always reduce to this case by enlarging the constant field of $F$;

- the class number of degree zero divisors $h^{0}(F)$ is prime with $p$, this is necessary to avoid characters of order $p$, but it is not too restrictive.

We extend all scalars to $W=\mathbb{Z}_{p}[\zeta]$, the Witt ring generated by a root of unity $\zeta$ of order $\left|G_{0}\right|$. Let $S=\{\mathfrak{p}, \infty\}$, let $\Theta_{\infty}(X)$ be the projection of the Stickelberger series $\Theta_{S}(X)$ to $\mathbb{Z}\left[\Gamma_{\infty} \times G_{0}\right] \llbracket X \rrbracket$ and write $\Theta_{\infty}(X, \chi)=\chi\left(\Theta_{\infty}\right)(X) \in W\left[\Gamma_{\infty}\right] \llbracket X \rrbracket$ as its $\chi$-part. We put

$$
\Theta_{\infty}^{\sharp}(X, \chi)= \begin{cases}\Theta_{\infty}(X, \chi) & \text { if } \chi \text { is of type } 1, \\ \frac{\Theta_{\infty}(X, \chi)}{1-X} & \text { if } \chi \text { is of type } 2 .\end{cases}
$$

Computing Fitting ideals for the $C_{n}$ and working our way through a limit process we prove

Theorem. (Theorems 5.21 and 5.22 Let $\chi$ be a character of type 1 or 2 . Then $C_{\infty}(\chi)$ is a finitely generated module over the Iwasawa algebra $\Lambda:=W \llbracket \Gamma_{\infty} \rrbracket$, and

$$
\operatorname{Fitt}_{\Lambda}\left(C_{\infty}(\chi)\right)=\left(\Theta_{\infty}^{\sharp}(1, \chi)\right) .
$$


This is what we call IMC for this setting, the relations with (special values of) $\mathfrak{p}$-adic $L$-functions are provided by the analytic interpolation properties mentioned before.

Remark 1.1. For the characters of type 3 we are only able to compute the Fitting ideal of a dual of $C_{n}$ and it is often non principal: we have no arithmetic interpretation (from the point of view of Iwasawa theory) for this situation yet so we decided to present it in a different paper (see [4, Section 3]).

\subsection{Setting and notations.}

- $F$ is a global function field of characteristic $p>0$, i.e. a finite algebraic extension of a field of transcendence degree 1 over a finite field $\mathbb{F}_{p^{r}}:=\mathbb{F}_{q}$ which we call the constant field of $F$. A more geometric interpretation would be to consider $F$ as the function field of a smooth projective curve $X$ defined over $\mathbb{F}_{q}$;

- $\infty$ is a fixed place of $F$ and $A$ is the subring of $F$ of the elements regular outside the place $\infty$;

- for any place $\nu$ of $F$ (including $\infty$ ), $F_{\nu}$ is the completion of $F$ at $\nu$. Its ring of integers will be denoted by $O_{\nu}$ and $U_{1}(\nu)$ will be the group of 1-units of $F_{\nu}$. The residue field $O_{\nu} /(\nu):=\mathbb{F}_{\nu}$ is a finite extension of $\mathbb{F}_{q}$ of degree $d_{\nu}:=\left[\mathbb{F}_{\nu}: \mathbb{F}_{q}\right]$ (also called the degree of $\nu$ ), its order will be denoted by $\mathbf{N} \nu:=q^{d_{\nu}}$;

- $v_{\nu}: F_{\nu} \rightarrow \mathbb{Z}$ is the (canonical) valuation at $\nu$ and $\pi_{\nu}$ will denote a fixed uniformizer for $F_{\nu}$, i.e. an element with $v_{\nu}\left(\pi_{\nu}\right)=1$;

- the degree of a fractional ideal $\mathfrak{a}=\prod_{\nu \neq \infty} \nu^{n_{\nu}}$ of $A$ is the quantity $\operatorname{deg}(\mathfrak{a})=\sum_{\nu \neq \infty} n_{\nu} d_{\nu}$.

\section{Stickelberger SERIES AND Artin $L$-FunCtion}

In this first section we introduce the main object of both the analytic and algebraic sides of the theory: the Stickelberger series. We provide here its convergence properties and its relation with complex Artin $L$-functions. In the subsequent sections it will appear as an incarnation of various $L$ or Zeta-functions and, on the algebraic side, as a generator for Fitting ideals of class groups.

2.1. Stickelberger series. Let $S$ be a nonempty finite set of places of $F$ that contains $\infty$ and denote by $F_{S}$ the maximal abelian extension of $F$ unramified outside $S$ and with $G_{S}:=\operatorname{Gal}\left(F_{S} / F\right)$ its Galois group. For every place $\nu \notin S$, let $\phi_{\nu}$ be the Frobenius at $\nu$, i.e. the unique element of $G_{S}$ that satisfies

$$
\phi_{\nu}(x) \equiv x^{\mathrm{N} \nu} \quad(\bmod \tilde{\nu})
$$

for every $x \in F_{S}$, where $\tilde{\nu}$ is any place of $F_{S}$ lying above $\nu$. The extension $F_{S} / F$ is unramified at $\nu$, so the decomposition group of $\nu$ in $G_{S}$ is pro-cyclic and topologically generated by $\phi_{\nu}$.

Definition 2.1. The Stickelberger series of $S$ is the power series defined by the Euler product

$$
\Theta_{S}(X)=\prod_{\nu \notin S}\left(1-\phi_{\nu}^{-1} X^{d_{\nu}}\right)^{-1} \in \mathbb{Z}\left[G_{S}\right] \llbracket X \rrbracket .
$$

For an equivalent formula, recall that every Euler factor $e_{\nu}(X):=1-\phi_{\nu}^{-1} X^{d_{\nu}}$ is invertible in $\mathbb{Z}\left[G_{S}\right] \llbracket X \rrbracket$ and let $\mathcal{I}_{S}$ be the set of fractional ideals of $A$ with support outside $S$ (recall 
$\infty \in S)$. Let $\phi_{\mathfrak{a}}$ be the Artin symbol for $\mathfrak{a} \in \mathcal{I}_{S}$, i.e. $\mathfrak{a}=\prod_{\nu \notin S} \nu^{n_{\nu}} \mapsto \phi_{\mathfrak{a}}=\prod_{\nu \notin S} \phi_{\nu}^{n_{\nu}}$, then

$$
\Theta_{S}(X)=\sum_{\mathfrak{a} \in \mathcal{I}_{S}, \mathfrak{a} \geqslant 0} \phi_{\mathfrak{a}}^{-1} X^{\operatorname{deg} \mathfrak{a}}=\sum_{n \geqslant 1}\left(\sum_{\substack{\mathfrak{a} \in \mathcal{I}_{S}, \mathfrak{a} \geqslant 0 \\ \operatorname{deg} \mathfrak{a}=n}} \phi_{\mathfrak{a}}^{-1}\right) X^{n}
$$

(where $\mathfrak{a} \geqslant 0$ denotes the integral ideals of $A$ ). For any $n$ there exists only a finite number of primes $\nu$ with degree equal to $n$, hence the series on the right is an element of $\mathbb{Z}\left[G_{S}\right] \llbracket X \rrbracket$.

2.2. Artin $L$-functions. Let $K / F$ be a finite subextension of $F_{S}$ whose Galois group is $G$ and let $S_{K} \subseteq S$ be the set of ramified places together with $\infty$ (in particular $S_{F}=\{\infty\}$ ).

For every prime $\nu$ let $\phi_{K, \nu} \in G$ be its Artin symbol: if $\nu \notin S$, then $\phi_{K, \nu}$ is the image of $\phi_{\nu}$ via the canonical projection $G_{S} \rightarrow G$, while for a ramified prime $\nu$, any lifting of its Frobenius in $G / I(\nu)$ (where $I(\nu)$ is the inertia at $\nu$ in $G$ ) will do. For every complex character $\chi$ of $G$, i.e. an element of $\operatorname{Hom}\left(G, \mathbb{C}^{\times}\right)$, we put $\chi(\nu)=\chi\left(\phi_{K, \nu}\right)$

Definition 2.2. The Artin $L$-function associated to $(K, \chi)$ is the complex variable function

$$
L_{K / F}(s, \chi)=\prod_{\nu \notin S_{K}}\left(1-\chi(\nu)(\mathbf{N} \nu)^{-s}\right)^{-1}, \quad \text { for } \mathfrak{R e}(s)>1
$$

(where the condition $\mathfrak{R e}(s)>1$ guarantees convergence).

Our goal in this section is to provide a link between $L_{K / F}(s, \chi)$ and $\Theta_{S}(X)$. In what follows we present formal equalities and avoid mentioning the radius of convergence: to be on the safe side one can always assume $\mathfrak{R e}(s)>1$.

Let $\Psi: G_{S} \rightarrow \mathbb{C}^{\times}$be a continuous character of $G_{S}$, i.e. a continuous homomorphism with respect to the natural topologies: it induces a ring homomorphism $\mathbb{Z}\left[G_{S}\right] \llbracket X \rrbracket \rightarrow \mathbb{C} \llbracket X \rrbracket$ (still denoted by $\Psi$ ).

Theorem 2.3. $\quad$ (a) Let $K / F, G$ and $\chi$ be as above, then there exists a continuous character $\Psi$ of $G_{S}$ such that

$$
\Psi\left(\Theta_{S}(X)\right)\left(q^{-s}\right)=L_{K / F}\left(s, \chi^{-1}\right) \prod_{\nu \in S-S_{K}}\left(1-\chi^{-1}(\nu) q^{-s d_{\nu}}\right) .
$$

(b) Let $\Psi$ be a continuous character of $G_{S}$. Then there exists $K \subset F_{S}$, finite over $F$ and a complex character $\chi$ of $\operatorname{Gal}(K / F)$ such that equation (2) holds.

Proof. (a) Let $\pi_{K}$ be the canonical projection $G_{S} \rightarrow G$ and put $\Psi:=\chi \circ \pi_{K}$, so that $\Psi\left(\phi_{\nu}^{-1}\right)=\chi^{-1}\left(\phi_{K, \nu}\right)$ for all $\nu \notin S$. Clearly $\Psi$ is a continuous character of $G_{S}$ and

$$
\begin{aligned}
\Psi\left(\Theta_{S}(X)\right)\left(q^{-s}\right) & =\prod_{\nu \notin S}\left(1-\chi^{-1}\left(\phi_{K, \nu}\right) q^{-s d_{\nu}}\right)^{-1} \\
& =L_{K / F}\left(s, \chi^{-1}\right) \prod_{\nu \in S-S_{K}}\left(1-\chi^{-1}(\nu) q^{-s d_{\nu}}\right) .
\end{aligned}
$$

(b) The profinite group $G_{S} / \operatorname{Ker}(\Psi)$ is topologically isomorphic to $\Psi\left(G_{S}\right)$ which is a compact subgroup of $\mathbb{C}^{\times}$, so $\operatorname{Ker}(\Psi)$ has finite index. Let $K$ be the fixed field of $\operatorname{Ker}(\Psi)$, so that $\operatorname{Gal}(K / F) \simeq G_{S} / \operatorname{Ker}(\Psi)$, and let $\chi$ be the character induced by $\Psi$ on this quotient. By definition $\Psi\left(\phi_{\nu}\right)=\chi\left(\phi_{K, \nu}\right)$ and

$$
\Psi\left(\Theta_{S}(X)\right)=\prod_{\nu \notin S}\left(1-\Psi\left(\phi_{\nu}^{-1}\right) X^{d_{\nu}}\right)^{-1}=\prod_{\nu \notin S}\left(1-\chi^{-1}\left(\phi_{K, \nu}\right) X^{d_{\nu}}\right)^{-1}
$$




$$
=\prod_{\nu \notin S_{K}}\left(1-\chi^{-1}(\nu) X^{d_{\nu}}\right)^{-1} \prod_{\nu \in S-S_{K}}\left(1-\chi^{-1}(\nu) X^{d_{\nu}}\right) .
$$

Equation (2) follows immediately evaluating at $X=q^{-s}$.

2.3. Stickelberger series and the Tate algebra. As an application of the previous theorem we prove that the Stickelberger series lies in the Tate algebra.

Let $\mathcal{R}$ be any topological ring. The Tate algebra $\mathcal{R}\langle X\rangle$ is the set of formal power series whose coefficients converge to 0 , in particular it contains the polynomial ring $\mathcal{R}[X]$. Let $\mathcal{O}$ be the ring of integers of a finite extension of $\mathbb{Q}_{p}$. We will be mainly interested in rings of the form $\mathcal{R}=\mathcal{O} \llbracket \Gamma \rrbracket$, where $\Gamma$ will be the Galois group of an infinite extension of function fields. We recall that the topology on this ring is the weakest such that the projection $\mathcal{O} \llbracket \Gamma \rrbracket \rightarrow \mathcal{O}[\operatorname{Gal}(K / F)]$ is continuous for each finite subextension $K / F$ : hence a sequence of elements $a_{n}$ of $\mathcal{O} \llbracket \Gamma \rrbracket$ converges to 0 if and only if the sequence of their projections is equal to 0 when $n$ is big enough, for each finite subextension $K / F$.

We consider $\Theta_{S}(X)$ as an element of $\mathcal{O}\left[G_{S}\right] \llbracket X \rrbracket$ via the (continuous) embedding $\mathbb{Z} \hookrightarrow \mathcal{O}$.

Proposition 2.4. The series $\Theta_{S}(X)$ is an element of the Tate algebra $\mathcal{O} \llbracket G_{S} \rrbracket\langle X\rangle$.

Proof. Since $(1-q X)^{-1}=\sum_{n \geqslant 0} q^{n} X^{n}$ is a unit in $\mathcal{O} \llbracket G_{S} \rrbracket\langle X\rangle$, it is enough to show that $f(X):=(1-q X) \Theta_{S}(X)$ is in the Tate algebra.

Let $\Psi: G_{S} \rightarrow \mathbb{C}^{\times}$be a continuous character. Following part (b) of Theorem 2.3 , let $K$ be the fixed field of $\operatorname{Ker}(\Psi)$ and $\chi$ the character induced by $\Psi$ on $\operatorname{Gal}(K / F)$ (note that, by definition, $\chi$ is trivial if and only if $\Psi$ is trivial on $G_{S}$, which leads to $K=F$ and $\left.S_{F}=\{\infty\}\right)$. Hence we have

$$
\Psi\left(\Theta_{S}(X)\right)\left(q^{-s}\right)=L_{K / F}\left(s, \chi^{-1}\right) \prod_{\nu \in S-S_{K}}\left(1-\chi^{-1}(\nu) q^{-s d_{\nu}}\right) .
$$

The full Artin $L$-function is defined by the Euler product on all primes $\nu$

$$
\widetilde{L}_{K / F}\left(s, \chi^{-1}\right)=\prod_{\nu}\left(1-\chi^{-1}(\nu)(\mathbf{N} \nu)^{-s}\right)^{-1}
$$

and differs from $L_{K / F}\left(s, \chi^{-1}\right)$ only for the factors associated to the primes of $S_{K}$. Thus

$$
\begin{aligned}
\Psi(f(X))\left(q^{-s}\right) & =\left(1-q^{1-s}\right) \Psi\left(\Theta_{S}(X)\right)\left(q^{-s}\right) \\
& =\left(1-q^{1-s}\right) \widetilde{L}_{K / F}\left(s, \chi^{-1}\right) \prod_{\nu \in S}\left(1-\chi^{-1}(\nu) q^{-s d_{\nu}}\right) .
\end{aligned}
$$

A theorem of Weil [31, $\mathrm{V}$, Théorèm 2.5] shows that if $\chi \neq \chi_{0}$, then $\widetilde{L}_{K / F}\left(s, \chi^{-1}\right)$ is a polynomial in $q^{-s}$, and, for $\chi=\chi_{0}$, we have

$$
\widetilde{L}_{K / F}\left(s, \chi_{0}\right)=\frac{P\left(q^{-s}\right)}{\left(1-q^{-s}\right)\left(1-q^{1-s}\right)},
$$

where $P(X)$ is a polynomial of degree $2 g-2(g$ is the genus of $F)$. Hence $\Psi(f(X))$ is a polynomial for each continuous character $\Psi$ (here we use the fact that $S$ is not empty): for each finite subextension $K / F$

$$
\pi_{K}(f(X)) \in \mathbb{Z}[\operatorname{Gal}(K / F)][X]
$$

(with $\pi_{K}$ the natural projection) and $f(X) \in \mathbb{Z} \llbracket G_{S} \rrbracket\langle X\rangle \subset \mathcal{O} \llbracket G_{S} \rrbracket\langle X\rangle$. 
In Section 5 we will need to evaluate the Stickelberger series $\Theta_{S}(X)$ at some element of $\mathcal{O} \llbracket G_{S} \rrbracket$, the previous proposition grants us that if we take $x$ in the unit disk $\left\{x \in \mathcal{O} \llbracket G_{S} \rrbracket\right.$ : $|x| \leqslant 1\}$, then $\Theta_{S}(x)$ converges.

\section{Stickelberger series And Goss Zeta-FunCtion}

We begin by giving a short description of the Goss Zeta-function and of its main properties (most notably its entireness): some results are known, we give references where avaliable and refer the reader to 12 for more details.

3.1. The Goss Zeta-function. Let $\mathbb{C}_{\infty}$ be the completion of a fixed algebraic closure of $F_{\infty}$ and put $\mathbb{S}_{\infty}:=\mathbb{C}_{\infty}^{\times} \times \mathbb{Z}_{p}$. The analogue of the Dedekind Zeta-function for $F$ has been originally defined for some special values (the integers) by Carlitz in [10] and later extended by Goss as a $\mathbb{C}_{\infty}$-valued function whose domain is $\mathbb{S}_{\infty}$ in [15]. This work of Goss may be interpreted as a sort of analytic continuation of the function defined by Carlitz. We give the definition and a summary of the main properties of the Goss Zeta-function, details and proofs can be found in [14, Chapter 8] or in [12, Sections 1.4 and 1.5].

Definition 3.1. A sign function on $F_{\infty}$ is any homomorphism $\operatorname{sgn}: F_{\infty}^{\times} \rightarrow \mathbb{F}_{\infty}^{\times}$such that its restriction to $\mathbb{F}_{\infty}^{\times}$is the identity. We extend sgn to all $F_{\infty}$ by defining $\operatorname{sgn}(0)=0$.

We fix a generator $\pi_{\infty}$ of the maximal ideal of $F_{\infty}$ and say that the sign function sgn is normalized if $\operatorname{sgn}\left(\pi_{\infty}\right)=1$. Since $U_{1}(\infty)$ is a pro- $p$-group and the image of sgn has order prime to $p$, every sign function is trivial on $U_{1}(\infty)$. The isomorphism

$$
F_{\infty}^{\times} \simeq \pi_{\infty}^{\mathbb{Z}} \times \mathbb{F}_{\infty}^{\times} \times U_{1}(\infty)
$$

allows us to write any $a \in F_{\infty}^{\times}$uniquely as

$$
a=\pi_{\infty}^{v_{\infty}(a)} \cdot \operatorname{sgn}(a) \cdot\langle a\rangle_{\infty} .
$$

We say an element $a \in F$ is positive if $\operatorname{sgn}(a)=1$ and denote by $A_{+}$the set of positive elements in $A$. Let $\mathcal{I}$ be the set of nonzero fractional ideals of $F$ and denote by $\mathcal{P}_{+}$the principal fractional ideals with a positive generator. The group $\mathcal{I} / \mathcal{P}_{+}$is finite and we put $h^{+}(A):=\left|\mathcal{I} / \mathcal{P}_{+}\right|:$it is easy to see that $h^{+}(A)=h^{0}(F) \cdot d_{\infty} \cdot\left(q^{d_{\infty}}-1\right) /(q-1)$. We recall that $d_{\infty}=\left[\mathbb{F}_{\infty}: \mathbb{F}_{q}\right]$ and, for any $a \in F_{\infty}^{\times}$, define the degree of $a$ as $\operatorname{deg}(a)=-d_{\infty} v_{\infty}(a)$. Note that if $I=(i)$ is principal, then the definition of $\operatorname{deg}(i)$ coincides with the degree of the ideal $I$, i.e. $\operatorname{deg}(i)=\operatorname{deg}(I):=|A / I|$.

For every $u \in U_{1}(\infty)$ and $y \in \mathbb{Z}_{p}$ the series $\sum_{n \geqslant 0}\left(\begin{array}{l}y \\ n\end{array}\right)(u-1)^{n}$ converges in $U_{1}(\infty)$, so we put

$$
u^{y}=((u-1)+1)^{y}:=\sum_{n \geqslant 0}\left(\begin{array}{l}
y \\
n
\end{array}\right)(u-1)^{n} .
$$

Definition 3.2. Given any ideal $I$, there exists a positive element $\alpha \in F^{\times}$such that $I^{h^{+}(A)}=(\alpha)$. Put $\langle I\rangle_{\infty}:=\langle\alpha\rangle_{\infty}^{1 / h^{+}(A)}$ as the unique $h^{+}(A)$-th root of $\langle\alpha\rangle_{\infty}$ which is still a 1-unit. Then, for any $s=(x, y) \in \mathbb{S}_{\infty}$, define

$$
I^{s}:=x^{\operatorname{deg}(I)}\langle I\rangle_{\infty}^{y} .
$$


Fix a $d_{\infty}$-th root of $\pi_{\infty}$ and call it $\pi_{*}$ : what follows will partly depend on this choice (but see statement (f) below). For every integer $j$ we put $s_{j}=\left(\pi_{*}^{-j}, j\right)$ : the map $j \mapsto s_{j}$ gives us an embedding $\mathbb{Z} \hookrightarrow \mathbb{S}_{\infty}$. It is easy to see that $\langle I\rangle_{\infty}$ and $I^{S}$ are well defined and belong to a finite extension of $F$ : we list a few fundamental properties of this exponential.

Proposition 3.3. For every positive $a, b \in F_{\infty}^{\times}, s, t \in \mathbb{S}_{\infty}, i, j \in \mathbb{Z}$ and every ideal $I \in \mathcal{I}$ one has

(a) $(a b)^{s}=a^{s} b^{s}$ and $a^{s+t}=a^{s} a^{t}$;

(b) $a^{s_{i}}=a^{i}$ and $\left(a^{s_{i}}\right)^{s_{j}}=a^{s_{i j}}$;

(c) $I^{s_{j}}$ is algebraic over $F$ and, if $I=(\alpha) \in \mathcal{P}_{+}$, then $I^{s}=\alpha^{s}$;

(d) let $F_{V}$ be the extension of $F$ obtained by adding every element of the form $I^{s_{1}}$, then $F_{V} / F$ is a finite extension with degree at most $h^{+}(A)$;

(e) let $F_{\infty, V}$ be the extension of $F_{\infty}$ obtained by adding every element of the form $\langle I\rangle_{\infty}$, then $F_{\infty, V} / F_{\infty}$ is a finite p-extension with degree dividing $h^{+}(A)$;

(f) if $F$ contains all the $d_{\infty}$-th roots of unity, then $F_{\boldsymbol{V}}$ does not depend on the choice of $\pi_{\infty}$ and $\pi_{*}$.

Proof. See [14, Sections 8.1, 8.2], other details can be found in [12, Section 1.4].

Definition 3.4. The Goss Zeta-function is defined by the sum

$$
\zeta_{A}(s)=\sum_{\mathfrak{a} \in \mathcal{I}, \mathfrak{a} \geqslant 0} \mathfrak{a}^{-s}=\sum_{n \geqslant 0}\left(\sum_{\begin{array}{c}
\mathfrak{a} \in \mathcal{I}, \mathfrak{a} \geqslant 0 \\
\operatorname{deg}(\mathfrak{a})=n
\end{array}}\langle\mathfrak{a}\rangle_{\infty}^{-y}\right) x^{-n}:=\sum_{n \geqslant 0} a_{n}(y) x^{-n}
$$

for $s=(x, y) \in \mathbb{S}_{\infty}$. It converges for $|x|_{\infty}>1$ and can also be rewritten as an Euler product

$$
\zeta_{A}(s)=\prod_{\nu \neq \infty}\left(1-\nu^{-s}\right)^{-1}=\prod_{\nu \neq \infty}\left(1-\langle\nu\rangle_{\infty}^{-y} x^{\left.-d_{\nu}\right)}\right)^{-1} .
$$

Estimates on the coefficients $a_{n}(y)$ allow to prove that the Goss Zeta-function is entire on $\mathbb{S}_{\infty}$ (in the sense of [14, Section 8.5]), i.e. the series provides an analytic continuation of $\zeta_{A}(s)$ to the whole $\mathbb{S}_{\infty}$ (for a sketch of the proof see [14, Sections 8.8 and 8.9], more details are in [12, Section 1.5]).

Theorem 3.5. (Analytic extension of the Goss Zeta-function) The serie $\zeta_{A}(s)$ is absolutely convergent for every $s=(x, y) \in \mathbb{S}_{\infty}$ and is also uniformly convergent on the compact subsets of $\mathbb{S}_{\infty}$.

3.2. Interpolation of the Goss Zeta-function. Let $W_{S}$ be the subgroup of $G_{S}$ generated by all Artin symbols $\phi_{\nu}$ with $\nu \notin S$ and let $K$ be the fixed field of the topological closure of $W_{S}$. Since $\phi_{\nu}$ is a topological generator of the decomposition group of $\nu$ in $G_{S}$, the extension $K / F$ is totally split at every prime $\nu \notin S$ and the Tchebotarev density theorem yields $K=F$, i.e. $G_{S}$ is the topological closure of $W_{S}$.

Lemma 3.6. Let $\lambda$ and $\mu$ be two distinct primes outside $S$, then $\phi_{\lambda} \neq \phi_{\mu}$.

Proof. This is just class field theory: consider the following subgroup of the idéles $\mathbb{I}_{F}$ of $F$

$$
H:=F_{\mu}^{\times} \times \prod_{\nu \neq \mu, \nu \notin S} O_{\nu}^{\times} \times \prod_{\nu \in S}\{1\},
$$


and let $K$ be the class field of $F^{\times} H$ (as usual $F^{\times}$is embedded diagonally in $\mathbb{I}_{F}$ ). Since $K / F$ is unramified outside $S$, totally split at $\mu$ and inert in $\lambda$, the decomposition groups of $\mu$ and $\lambda$ in $G_{S}$ do not coincide and their Artin symbols are distinct.

Let $f:=\left[F_{\infty, \mathbf{V}}: F_{\infty}\right]$ (recall that $f$ is a power of $p$ by Proposition 3.3 part (e)) and let $N: F_{\infty, \mathbf{V}}^{\times} \rightarrow F_{\infty}^{\times}$be the norm map. For any $y \in \mathbb{Z}_{p}$ and any $\nu \notin S$ we define

$$
\Psi_{y}\left(\phi_{\nu}\right)=N\left(\langle\nu\rangle_{\infty}^{-1}\right)^{y / f} .
$$

This is well defined because the norm sends 1-units to 1-units and it is possible to take the $f$-th root without ambiguity.

Lemma 3.7. The map $\Psi_{y}$ extends to a group homomorphism $\Psi_{y}: G_{S} \rightarrow \mathbb{C}_{\infty}^{\times}$and induces $a \mathbb{C}_{\infty}^{\times}$-character on $\mathbb{I}_{F}$.

Proof. Since $W_{S}$ is generated by the Artin symbols, we just put

$$
\Psi_{y}(\sigma)=\Psi_{y}\left(\prod_{\nu \notin S} \phi_{\nu}^{n_{\nu}}\right)=\prod_{\nu \notin S} \Psi_{y}\left(\phi_{\nu}\right)^{n_{\nu}} .
$$

Now $\Psi_{y}: W_{S} \rightarrow \mathbb{C}_{\infty}^{\times}$is a continuous homomorphism (as composition of continuous maps) and it can be extended to the topological closure $G_{S}$. It is well defined (by Lemma 3.6) and can be extended to idéles via the natural injective map

$$
\varphi: \mathcal{I} \longrightarrow \mathbb{I}_{F} \text {, given by } \varphi(\mathfrak{a})=\prod_{\nu} \pi_{\nu}^{v_{\nu}(\mathfrak{a})},
$$

and the reciprocity map $\operatorname{rec}_{S}: \mathbb{I}_{F} \rightarrow G_{S}$. Since $\operatorname{Ker}\left(\operatorname{rec}_{S}\right)=F^{\times} \cdot \prod_{\nu \notin S} O_{\nu}^{\times}$, the map $\operatorname{rec}_{S} \circ \varphi$ is still injective. Obviously $\sigma=\prod_{\nu \notin S} \phi_{\nu}^{n_{\nu}} \in W_{S}$ is $\left(\operatorname{rec}_{S} \circ \varphi\right)\left(\prod_{\nu \notin S} \nu^{n_{\nu}}\right)=\left(\operatorname{rec}_{S} \circ \varphi\right)(\mathfrak{a})$ for some fractional ideal $\mathfrak{a}$ in $\mathcal{I}$, and $\Psi_{y}(\sigma)$ does not depend on the chosen expression for $\sigma$.

We mention that the interpretation of $\Psi_{y}$ as a $\mathbb{C}_{\infty}^{\times}$-character on $S$-idéles is the approach suggested in [2, Theorem 3.8 and Remark 3.9]: we shall see a more explicit relation between $\Psi_{y}$ and $\operatorname{rec}_{S}$ in the special case presented in the next section.

The extension $\Psi_{y}: \mathbb{Z}\left[G_{S}\right] \llbracket X \rrbracket \rightarrow \mathbb{C}_{\infty} \llbracket X \rrbracket$ gives an interpolation formula for the Goss Zetafunction (the case of the Carlitz cyclotomic extension is presented in [4, Theorem 4.2]).

Theorem 3.8. For every $s=(x, y) \in \mathbb{S}_{\infty}$ we have

$$
\Psi_{y}\left(\Theta_{S}(X)\right)(x)=\zeta_{A}(-s) \prod_{\nu \in S, \nu \neq \infty}\left(1-\nu^{s}\right) .
$$

Proof. Let $\nu$ be a prime outside $S$, assume $[\nu]$ has order $n$ in $\mathcal{I} / \mathcal{P}_{+}$and let $\alpha$ be a positive element such that $\nu^{n}=(\alpha)$. By definition $\langle\nu\rangle_{\infty}^{n}=\langle\alpha\rangle_{\infty}$. Now write $n=p^{r} n^{\prime}$ with $\left(p, n^{\prime}\right)=1$ and let $u \in U_{1}(\infty)$ be the only 1-unit verifying $u^{n^{\prime}}=\langle\alpha\rangle_{\infty}$, so that $\langle\nu\rangle_{\infty}$ is a root of $f(X)=X^{p^{r}}-u \in F_{\infty}[X]$. Let $g(X)$ be the minimal polynomial of $\langle\nu\rangle_{\infty}$ over $F_{\infty}$. Since $f(X)$ is totally inseparable, it must be $f(X)=g(X)^{p^{l}}$ and $g(X)=X^{p^{k}}-v$, where $l, k$ and $v$ satisfy $r=k+l$ and $u=v^{p^{l}}$. Then $F_{\infty}\left(\langle\nu\rangle_{\infty}\right) / F_{\infty}$ has degree $p^{k}$, while $F_{\infty, \mathbf{V}} / F_{\infty}\left(\langle\nu\rangle_{\infty}\right)$ has degree $f / p^{k}$. Therefore

$$
N\left(\langle\nu\rangle_{\infty}\right)=N_{F_{\infty}\left(\langle\nu\rangle_{\infty}\right) / F_{\infty}}\left(\langle\nu\rangle_{\infty}\right)^{f / p^{k}}=v^{f / p^{k}}=\langle\nu\rangle_{\infty}^{f}
$$


(everything works for $p=2$ as well since in that case $v=-v$ ).

Hence $\Psi_{y}\left(\phi_{\nu}\right)=\langle\nu\rangle_{\infty}^{-y}$ and

$$
\begin{aligned}
\Psi_{y}\left(\Theta_{S}(X)\right)(x) & =\prod_{\nu \notin S}\left(1-\Psi_{y}\left(\phi_{\nu}^{-1}\right) x^{d_{\nu}}\right)^{-1}=\prod_{\nu \notin S}\left(1-\langle\nu\rangle_{\infty}^{y} x^{d_{\nu}}\right)^{-1} \\
& =\zeta_{A}(-s) \prod_{\nu \in S, \nu \neq \infty}\left(1-\nu^{s}\right) .
\end{aligned}
$$

3.3. A special case. Here we provide a link between $\Psi_{y}$ and the Artin reciprocity map assuming that the class number of $F$ is 1 and that $d_{\infty}=1$ (the hypotheses we shall use in Section 5): in particular $\mathbb{F}_{\infty}=\mathbb{F}_{q}$ and every element of $F^{\times}$can be written uniquely as product of a constant and a positive element. We choose an uniformizer at $\infty$ by simply taking a prime $\mathfrak{p} \neq \infty$ of degree 1 , letting $\pi_{\mathfrak{p}}$ be its unique positive generator and then putting $\pi_{\infty}:=\pi_{\mathfrak{p}}^{-1}$. Note that this uniformizer is positive and is an element of $F^{1}$. Finally note that, in the notations of Proposition 3.3, $F_{\mathbf{V}}=F$ and $F_{\infty, \mathbf{V}}=F_{\infty}$ : therefore $\Psi_{y}\left(\phi_{\nu}\right)=\left\langle\pi_{\nu}\right\rangle_{\infty}^{-y}$ belongs to $U_{1}(\infty)$.

Theorem 3.9. Let $F_{\infty,+}^{\times}$be the kernel of the sign function. The natural inclusion

$$
F_{\infty,+}^{\times} \times \prod_{\nu \neq \infty} O_{\nu}^{\times}=: \mathcal{H} \hookrightarrow \mathbb{I}_{F}
$$

induces an isomorphism $\mathcal{H} \simeq \mathbb{I}_{F} / F^{\times}$.

Proof. Let $\mathbf{i}=\left(i_{\infty}, i_{\nu_{1}}, i_{\nu_{2}}, \ldots\right)$ and $\mathbf{j}=\left(j_{\infty}, j_{\nu_{1}}, j_{\nu_{2}}, \ldots\right)$ be two idéles in $\mathcal{H}$ with the same image in $\mathbb{I}_{F} / F^{\times}$, i.e. there is an $x \in F^{\times}$such that $\mathbf{i}=x \mathbf{j}$. For every $\nu \neq \infty, i_{\nu}=x j_{\nu}$ yields $v_{\nu}(x)=0$ because $i_{\nu}$ and $j_{\nu}$ are units. Moreover the product formula implies $v_{\infty}(x)=-\sum_{\nu \neq \infty} d_{\nu} v_{\nu}(x)=0$ and so $x$ is a constant. Finally since $i_{\infty}=x j_{\infty}$ and both $i_{\infty}$ and $j_{\infty}$ are positive, we have $x=1$.

To complete the proof we need surjectivity: let $\mathbf{i}=\left(i_{\infty}, i_{\nu_{1}}, i_{\nu_{2}}, \ldots\right) \in \mathbb{I}_{F}$ and put

$$
x_{\mathbf{i}}=\operatorname{sgn}\left(i_{\infty}\right)^{-1} \prod_{\nu \neq \infty} \pi_{\nu}^{-v_{\nu}\left(i_{\nu}\right)} \in F^{\times} .
$$

It is easy to check that the idéle $x_{\mathbf{i}} \mathbf{i}$ is in $\mathcal{H}$, and the proof is complete.

Let $C_{F}:=\mathbb{I}_{F} / F^{\times}$and consider the subgroup $O_{S}=\prod_{\nu \notin S} O_{\nu}^{\times} / F^{\times}$: by class field theory, the Artin map induces a continuous embedding $\operatorname{rec}_{S}: C_{F} / O_{S} \rightarrow G_{S}$, which is not surjective because $G_{S}$ is profinite, while the quotient $C_{F} / O_{S}$ is not. Let $\overline{C_{F} / O_{S}}$ be the profinite completion of $C_{F} / O_{S}$, then the map $\operatorname{rec}_{S}$ extends to an isomorphism of topological groups $\widehat{\operatorname{rec}}_{S}: \widehat{C F}_{F} / O_{S} \stackrel{\sim}{\longrightarrow} G_{S}$ and, by Theorem 3.9 , one has the isomorphism

$$
\widehat{C_{F} / O_{S}} \simeq \widehat{\left\langle\pi_{\infty}\right\rangle} \times U_{1}(\infty) \times \prod_{\substack{\nu \in S \\ \nu \neq \infty}} O_{\nu}^{\times}
$$

where $\widehat{\left\langle\pi_{\infty}\right\rangle} \simeq \widehat{\mathbb{Z}}$. We denote by $\widehat{\pi}$ the canonical projection $\widehat{C_{F} / O_{S}} \rightarrow U_{1}(\infty)$.

\footnotetext{
${ }^{1}$ If $F$ is the rational function field $\mathbb{F}_{q}(t)$, and $\infty=\frac{1}{t}$, we are simply taking as uniformizer the element $\pi_{\infty}=1 /(t-\alpha)$, where $\alpha$ is any element of $\mathbb{F}_{q}^{\times}$and $\mathfrak{p}=(t-\alpha)$.
} 
Theorem 3.10. For every $y \in \mathbb{Z}_{p}$ we have the following commutative diagram

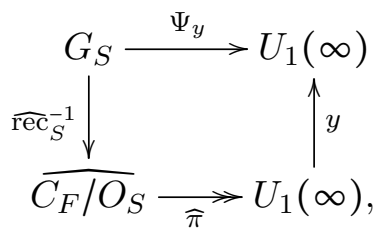

where $y$ denotes the raise-to-the-power $y$ map.

Proof. We prove the case $y=1$ first. Since $G_{S}$ is the topological closure of $W_{S}$, and all maps are continuous, it is enough to show that $\Psi_{1}\left(\phi_{\nu}\right)=\widehat{\pi} \circ \widehat{r e c}_{S}^{-1}\left(\phi_{\nu}\right)$ for every $\nu \notin S$.

The local Artin map $\operatorname{rec}_{\nu}$ sends $\pi_{\nu}$ to $\phi_{\nu}$, because the extension $F_{S} / F$ is unramified at $\nu$. Let $\mathbf{i}_{\nu}$ be the idéle whose $\nu$-coordinate is equal to $\pi_{\nu}$ and whose $\mu$-coordinates for $\mu \neq \nu$ are all equal to 1 , and let $\left[\mathbf{i}_{\nu}\right] \in C_{F}$ be its equivalence class. Then

$$
\phi_{\nu}=\operatorname{rec}_{S}\left(\left[\mathbf{i}_{\nu}\right]\right)=\operatorname{rec}_{S}\left(\left[\pi_{\nu}^{-1} \mathbf{i}_{\nu}\right]\right)
$$

and, noting that $\pi_{\nu}^{-1} \mathbf{i}_{\nu} \in \mathcal{H}$, we obtain

$$
\widehat{\pi} \circ \widehat{\operatorname{rec}}_{S}^{-1}\left(\phi_{\nu}\right)=\left\langle\pi_{\nu}^{-1}\right\rangle_{\infty}=\Psi_{1}\left(\phi_{\nu}\right) \text {. }
$$

For a general $y \in \mathbb{Z}_{p}$ note that, for any Artin symbol $\phi_{\nu}$, we have $\Psi_{y}\left(\phi_{\nu}\right)=\Psi_{1}\left(\phi_{\nu}\right)^{y}$.

\section{Stickelberger SERIES AND $\nu$-ADIC Zeta-FunCtions}

Fix a place $\nu$ different from $\infty$ : in this section we consider a $\nu$-adic analogue of the Goss Zeta-function. Let $F_{\nu}, \mathbb{C}_{\nu}, \mathbb{F}_{\nu}$ and $\pi_{\nu}$ be the $\nu$-adic versions of the objects defined for the place $\infty$. Fix an algebraic closure $\bar{F}$ of $F$ and let $\iota: \bar{F} \leftrightarrow \mathbb{C}_{\nu}$ be an $F$-embedding that is the identity on the compositum of the algebraic closure of $\mathbb{F}_{q}$ and $F_{\mathbf{V}}$. All the objects that we define here depend on $\iota$, but we will omit this dependency to simplify notations.

The field $F_{\nu, \mathbf{V}}:=\iota\left(F_{\mathbf{V}}\right) F_{\nu}$ will play the role of $F_{\infty, \mathbf{V}}$ : as in Proposition 3.3, one can show that $F_{\nu, \mathbf{V}}$ is a $p$-extension of $F_{\nu}$ with degree dividing $h^{+}(A)$. The residue field of $F_{\nu, \mathbf{V}}$ is still $\mathbb{F}_{\nu}$ and the cyclic group $\mathbb{Z} /\left|\mathbb{F}_{\nu}^{\times}\right|$acts on its multiplicative subgroup. Let $\mathbb{S}_{\nu}=\mathbb{C}_{\nu}^{\times} \times \mathbb{Z}_{p} \times \mathbb{Z} /\left|\mathbb{F}_{\nu}^{\times}\right|$, which is a subgroup of the group of $\mathbb{C}_{\nu}^{\times}$-valued characters on $F_{\nu}^{\times}$, and take $s_{\nu}=(x, y, j) \in \mathbb{S}_{\nu}$ : we have to define the exponential $I^{s_{\nu}} \in \mathbb{C}_{\nu}^{\times}$, for every fractional ideal $I \in \mathcal{I}$ coprime with $\nu$.

For $s_{1}=\left(\pi_{*}^{-1}, 1\right) \in \mathbb{S}_{\infty}$ the element $I^{s_{1}} \in F_{\mathbf{V}}$ is a root of the polynomial $X^{h^{+}(A)}-\alpha$, where $\alpha$ is the unique positive generator of $I^{h^{+}(A)}$, so the valuation at $\nu$ of $I^{s_{1}}$ is equal to zero. Hence $\iota\left(I^{s_{1}}\right)$ is a unit in $F_{\nu, \mathbf{V}}$ and can be written uniquely as a product

$$
\iota\left(I^{s_{1}}\right)=\omega(I)\langle I\rangle_{\nu},
$$

for some $\omega(I) \in \mathbb{F}_{\nu}^{\times}$and $\langle I\rangle_{\nu}$ a 1-unit of $F_{\nu, \mathbf{V}}$. With the above notation, it is easy to check that the map $\omega: \mathcal{I}_{\nu} \rightarrow \mathbb{F}_{\nu}^{\times}$sending $I$ to $\omega(I)$ is a group homomorphism on the group $\mathcal{I}_{\nu}$ of fractional ideals prime with $\nu$ (it is basically a Teichmüller character).

Finally for $s_{\nu}=(x, y, j) \in \mathbb{S}_{\nu}$ and $I \in \mathcal{I}_{\nu}$ we define

$$
I^{s_{\nu}}=x^{\operatorname{deg}(I)} \omega(I)^{j}\langle I\rangle_{\nu}^{y} .
$$

We can embed $\mathbb{Z}$ in $\mathbb{S}_{\nu}$ via the map $j \in \mathbb{Z} \mapsto s_{\nu, j}=(1, j, j) \in \mathbb{S}_{\nu}$ and one can show that this $\nu$-adic exponential satisfies properties analogous to the ones of Proposition 3.3 . 
Proposition 4.1. For every $s_{\nu}, t_{\nu} \in \mathbb{S}_{\nu}, I, J \in \mathcal{I}_{\nu}$ and $i, j \in \mathbb{Z}$, one has

- $I^{s_{\nu}+t_{\nu}}=I^{s_{\nu}} I^{t_{\nu}}$ and $(I J)^{s_{\nu}}=I^{s_{\nu}} J^{s_{\nu}}$.

- $\left(I^{s_{\nu, i}}\right)^{s_{\nu, j}}=I^{s_{\nu, i j}}$ and $I^{s_{\nu, j}}=I^{s_{j}}$. In particular $I^{s_{\nu, j}}$ is algebraic over $F$.

Definition 4.2. The $\nu$-adic Goss Zeta-function is defined as

$$
\zeta_{\nu}\left(s_{\nu}\right)=\sum_{\mathfrak{a} \in \mathcal{I}_{\nu}, \mathfrak{a} \geqslant 0} \mathfrak{a}^{-s_{\nu}}=\prod_{\mathfrak{p} \neq \nu, \infty}\left(1-\mathfrak{p}^{-s_{\nu}}\right)^{-1} .
$$

4.1. Interpolation of the $\nu$-adic Zeta-function. We look for an analogue of Theorem 3.8 for $\zeta_{\nu}\left(s_{\nu}\right)$ : this will be done only for primes $\nu \in S-\{\infty\}$, so for the rest of this section we take such a prime $\nu$ assuming $\# S \geqslant 2$. Let $f_{\nu}$ be the degree of the extension $F_{\nu, \mathbf{V}} / F_{\nu}$ and denote by $N_{\nu}: F_{\nu, \mathbf{V}}^{\times} \rightarrow F_{\nu}^{\times}$the norm map. Take $(y, j) \in \mathbb{Z}_{p} \times \mathbb{Z} /\left|\mathbb{F}_{\nu}^{\times}\right|$and, for $\mathfrak{p} \notin S$, put

$$
\Psi_{y, j}\left(\phi_{\mathfrak{p}}\right)=N_{\nu}\left(\langle\mathfrak{p}\rangle_{\nu}^{-1}\right)^{y / f_{\nu}} \omega(\mathfrak{p})^{-j},
$$

which is well defined by Lemma 3.6. Adapting the proof of Lemma 3.7 one obtains

Lemma 4.3. For every $(y, j) \in \mathbb{Z}_{p} \times \mathbb{Z} /\left|\mathbb{F}_{\nu}^{\times}\right|$the map $\Psi_{y, j}$ extends to a continuous ring homomorphism $\mathbb{Z}\left[G_{S}\right] \llbracket X \rrbracket \rightarrow \mathbb{C}_{\nu} \llbracket X \rrbracket$.

Here is the relation between Stickelberger series and the $\nu$-adic Zeta-function.

Theorem 4.4. For every $s_{\nu}=(x, y, j) \in \mathbb{S}_{\nu}$ (with $\nu \in S-\{\infty\}$ ) we have

$$
\Psi_{y, j}\left(\Theta_{S}(X)\right)(x)=\zeta_{\nu}\left(-s_{\nu}\right) \prod_{\substack{\mathfrak{p} \in S \\ \mathfrak{p} \neq \nu, \infty}}\left(1-\mathfrak{p}^{s_{\nu}}\right) .
$$

Proof. Le $\mathfrak{p}$ be a prime not in $S, n$ the exact order of $[\mathfrak{p}]$ in $\mathcal{I} / \mathcal{P}_{+}$and $\alpha$ a positive element such that $\mathfrak{p}^{n}=(\alpha)$. We have $\left(\mathfrak{p}^{n}\right)^{s_{1}}=(\alpha)^{s_{1}}=\alpha$ and so

$$
\omega(\mathfrak{p})^{n}\langle\mathfrak{p}\rangle_{\nu}^{n}=\iota\left(\mathfrak{p}^{s_{1}}\right)^{n}=\iota(\alpha)^{s_{1}}=\alpha,
$$

which leads to $\langle\mathfrak{p}\rangle_{\nu}^{n}=\langle\alpha\rangle_{\nu}$.

Proceeding like in Theorem 3.8 , one finds that $F_{\nu, \mathbf{V}} / F_{\nu}\left(\left\langle\mathfrak{p}_{\nu}\right\rangle\right)$ has degree $f_{\nu} / p^{k}$ and

$$
N_{\nu}\left(\langle\mathfrak{p}\rangle_{\nu}\right)=N_{K / F_{\nu}}\left(\langle\mathfrak{p}\rangle_{\nu}\right)^{f_{\nu} / p^{k}}=\langle\mathfrak{p}\rangle_{\nu}^{f_{\nu}} .
$$

From this we obtain that $\Psi_{y, j}\left(\phi_{\mathfrak{p}}\right)=\omega(\mathfrak{p})^{-j}\langle\mathfrak{p}\rangle_{\nu}^{-y}$ and

$$
\Psi_{y, j}\left(\Theta_{S}(X)\right)=\prod_{\mathfrak{p} \notin S}\left(1-\Psi_{y, j}\left(\phi_{\mathfrak{p}}^{-1}\right) X^{d_{\mathfrak{p}}}\right)^{-1}=\prod_{\mathfrak{p} \notin S}\left(1-\omega(\mathfrak{p})^{j}\langle\mathfrak{p}\rangle_{\nu}^{y} X^{d_{\mathfrak{p}}}\right)^{-1} .
$$

Hence

$$
\Psi_{y, j}\left(\Theta_{S}(X)\right)(x)=\prod_{\mathfrak{p} \notin S}\left(1-\omega(\mathfrak{p})^{j}\langle\mathfrak{p}\rangle_{\nu}^{y} x^{d_{\mathfrak{p}}}\right)^{-1}=\zeta_{\nu}\left(-s_{\nu}\right) \prod_{\substack{\mathfrak{p} \in S \\ \mathfrak{p} \neq \nu, \infty}}\left(1-\mathfrak{p}^{s_{\nu}}\right) .
$$

4.2. Interpolation via a $\nu$-adic $L$-function. We investigate the values of $\nu$-adic Zetafunctions at integers, introducing $\nu$-adic $L$-series to interpolate them. This is one of the main features of Iwasawa theory, where $\mathfrak{p}$-adic $L$-functions (for $\mathfrak{p}$ a prime in a global field) usually represent the analytic counterpart of characteristic ideals of Iwasawa modules.

We shall use the following (see [14, Lemma 8.8.1] or [32, Theorem 5.1.2]). 
Lemma 4.5. Let $K$ be a function field with constant field $\mathbb{F}_{q}, v$ any normalized valuation on $K$ and $W \subset K$ an $\mathbb{F}_{q}$-vector space with finite dimension. Assume that $v(w)>0$ for every $w \in W$. If $i$ is an integer with $0 \leqslant i<(q-1) \operatorname{dim}_{\mathbb{F}_{q}} W$, then for every $x \in K$ we have

$$
\sum_{w \in W}(x+w)^{i}=0 \text {. }
$$

For each pair of non negative integers $j$ and $n$, we define

$$
S_{n}(j)=\sum_{\mathfrak{a} \geqslant 0, \operatorname{deg} \mathfrak{a}=n} \mathfrak{a}^{s_{j}} \quad \text { and } \quad Z(X, j)=\sum_{n \geqslant 0} S_{n}(j) X^{n} \in F_{\mathbf{V}} \llbracket X \rrbracket .
$$

Lemma 4.6. The $Z(X, j)$ are polynomials of degree less than or equal to $d_{\infty}+2 g+\left\lfloor\frac{j}{q-1}\right\rfloor$.

Proof. Fix a non negative integer $j$ and an $n>d_{\infty}+2 g+\left\lfloor\frac{j}{q-1}\right\rfloor$.

For any $\ell=1, \ldots, h^{+}$, fix a representative $\mathfrak{a}_{\ell} \in \mathcal{I}$ for the class $C_{\ell}$ of $\mathcal{I} / \mathcal{P}_{+}$. Then

$$
S_{n}(j)=\sum_{j=1}^{h^{+}} \sum_{\substack{\mathfrak{a} \geqslant 0, \mathfrak{a} \in C_{\ell} \\
\operatorname{deg}(\mathfrak{a})=n}} \mathfrak{a}^{s_{j}}=\sum_{j=1}^{h^{+}} \mathfrak{a}_{\ell}^{s_{j}} \cdot \sum_{\begin{array}{c}
\alpha \in F_{+}, \alpha \mathfrak{a}_{\ell} \geqslant 0 \\
\operatorname{deg}(\alpha)=n-\operatorname{deg}\left(\mathfrak{a}_{\ell}\right)
\end{array}} \alpha^{j}:=\sum_{j=1}^{h^{+}} S_{n}\left(C_{\ell}, j\right) .
$$

To prove $S_{n}\left(C_{\ell}, j\right)=0$, we put $n_{\ell}=n-\operatorname{deg}\left(\mathfrak{a}_{\ell}\right)$ and only consider the case $d_{\infty} \mid n_{\ell}$, otherwise the claim is trivial. Let $\mathcal{X}:=\left\{\alpha \in F_{+}: \operatorname{deg}(\alpha)=n_{\ell}\right.$ and $\left.\alpha \mathfrak{a}_{\ell} \geqslant 0\right\}$, denote by $D_{1}$ the divisor $\operatorname{Div}\left(\mathfrak{a}_{\ell}\right)+\left(n_{\ell} / d_{\infty}-1\right) \infty$ and by $\mathcal{L}\left(D_{1}\right):=\left\{\alpha \in F^{\times}: \operatorname{Div}(\alpha)+D_{1} \geqslant 0\right\}$ the associated Riemann-Roch space. The set $\mathcal{L}\left(D_{1}\right)$ acts freely by traslation on $\mathcal{X}$ : write $\mathcal{X}$ as the union of $r$ orbits $\mathcal{X}_{l}$ and fix a representative $x_{l} \in \mathcal{X}_{l}$. Then

$$
S_{n}\left(C_{\ell}, j\right)=\mathfrak{a}_{\ell}^{s_{j}} \sum_{l=1}^{r} \sum_{u \in \mathcal{L}\left(D_{1}\right)}\left(u+x_{l}\right)^{j}=\mathfrak{a}_{\ell}^{s_{j}} \sum_{l=1}^{r} x_{l}^{j} \sum_{w \in x_{l}^{-1} \mathcal{L}\left(D_{1}\right)}(w+1)^{j} .
$$

We have $v_{\infty}\left(x_{l}\right)=-n_{\ell} / d_{\infty}$ and $v_{\infty}(u) \geqslant 1-n_{\ell} / d_{\infty}$. Therefore $v_{\infty}(w)$ is positive for every $w \in x_{l}^{-1} \mathcal{L}\left(D_{1}\right)$ and the vector space $x_{l}^{-1} \mathcal{L}\left(D_{1}\right)$ satisfies the hypotheses of Lemma 4.5. Hence the inner sum is zero when $j<(q-1) \operatorname{dim}_{\mathbb{F}_{q}} \mathcal{L}\left(D_{1}\right)=(q-1)\left(n-d_{\infty}-g+1\right)$ (the last equality comes from the Riemann-Roch theorem).

The polynomials $Z(X, j)$ are strictly related to the special values of the Goss Zetafunction since we have that $Z(1, j)=\zeta_{A}\left(-s_{j}\right)$ for any $j \in \mathbb{N}$. We provide here a $\nu$-adic interpolation of this polynomials, which will be linked to the special values of the $\nu$-adic Goss Zeta-function.

Definition 4.7. For any $y \in \mathbb{Z}_{p}$ and $i \in \mathbb{Z} /\left|\mathbb{F}_{\nu}^{*}\right|$, the $\nu$-adic $L$-series is defined by

$$
L_{\nu}\left(X, y, \omega^{i}\right)=\sum_{n \geqslant 0}\left(\sum_{\substack{\mathfrak{a} \in \mathcal{I}_{\nu}, \mathfrak{a} \geqslant 0 \\ \operatorname{deg}(\mathfrak{a})=n}} \omega(\mathfrak{a})^{i}\langle\mathfrak{a}\rangle_{\nu}^{y}\right) X^{n} .
$$

Proposition 4.8. For every $s_{\nu}=(x, y, i) \in \mathbb{S}_{\nu}$ we have

$$
L_{\nu}\left(x, y, \omega^{i}\right)=\zeta_{\nu}\left(-s_{\nu}\right) .
$$

Proof. For a fractional ideal $\mathfrak{a}$ coprime with $\nu$, we have $\mathfrak{a}^{s_{\nu}}=\omega(\mathfrak{a})^{i}\langle\mathfrak{a}\rangle_{\nu}^{y} x^{\operatorname{deg} \mathfrak{a}}$, so

$$
L_{\nu}\left(x, y, \omega^{i}\right)=\sum_{n \geqslant 0} \sum_{\substack{\mathfrak{a} \in \mathcal{I}_{\nu}, \mathfrak{a} \geqslant 0 \\ \operatorname{deg}(\mathfrak{a})=n}} \omega(\mathfrak{a})^{i}\langle\mathfrak{a}\rangle_{\nu}^{y} x^{n}
$$




$$
=\sum_{n \geqslant 0} \sum_{\substack{\mathfrak{a} \in \mathcal{I}_{\nu}, \mathfrak{a} \geqslant 0 \\ \operatorname{deg}(\mathfrak{a})=n}} \mathfrak{a}^{s_{\nu}}=\sum_{\mathfrak{a} \in \mathcal{I}_{\nu}, \mathfrak{a} \geqslant 0} \mathfrak{a}^{s_{\nu}}=\zeta_{\nu}\left(-s_{\nu}\right) .
$$

The previous proposition, Theorem 4.4 and Section 2.3 show that the function $L_{\nu}\left(X, y, \omega^{i}\right)$ converges on the closed unit disc: since our application to class groups will only require specialization at $X=1$ we do not deal with its entireness here (which anyway can be proved just like the entireness of $\left.\zeta_{A}\right)$.

The relation with the polynomials $Z(X, j)$ for some particular values of $i$ and $j$ can be made more explicit: in particular the following theorem shows that, for some $i$ and $j$, the series $L_{\nu}\left(X, y, \omega^{i}\right)$ is actually a polynomial. Computations will shift between $\infty$ and $\nu$ using formulas seen in Section 3.2 .

Theorem 4.9. Assume that $\nu \in S-\{\infty\}$.

(a) Let $i$ and $j$ be two non negative integers, such that $i \equiv j\left(\bmod q^{d_{\nu}}-1\right)$, then

$$
L_{\nu}\left(X, j, \omega^{i}\right)=Z(X, j)\left(1-\nu^{s_{j}} X^{d_{\nu}}\right) .
$$

In particular $L_{\nu}\left(X, j, \omega^{i}\right)$ in a polynomial.

(b) For every $y \in \mathbb{Z}_{p}$ we have

$$
L_{\nu}\left(X, y, \omega^{i}\right) \equiv Z(X, i) \quad(\bmod \bar{\nu}),
$$

where $\bar{\nu}$ denotes any prime of $F_{\boldsymbol{V}}$ lying above $\nu$.

Proof. (a) Using the unique factorization of ideals in $A$ and the multiplicativity on $\mathcal{I}$ of the maps $I \mapsto\langle I\rangle_{\nu}$ and $I \mapsto \omega(I)$ one rewrites

$$
L_{\nu}\left(X, j, \omega^{i}\right)=\prod_{\mathfrak{p} \neq \nu, \infty}\left(1-\omega(\mathfrak{p})^{i}\langle\mathfrak{p}\rangle_{\nu}^{j} X^{d_{\mathfrak{p}}}\right)^{-1}
$$

In the proof of Theorem 4.4 we have seen that this product is equal to

$$
\Psi_{j, i}\left(\Theta_{S}(X)\right) \cdot \prod_{\substack{\mathfrak{p} \in S \\ \mathfrak{p} \neq \infty, \nu}}\left(1-\omega(\mathfrak{p})^{i}\langle\mathfrak{p}\rangle_{\nu}^{j} X^{d_{\mathfrak{p}}}\right)^{-1} .
$$

Since $i \equiv j\left(\bmod q^{d_{\nu}}-1\right)$ and $\iota$ is the identity on $\mathfrak{p}^{s_{1}} \in F_{\mathbf{V}}$, one has

$$
\Psi_{j, i}\left(\phi_{\mathfrak{p}}^{-1}\right)=\omega(\mathfrak{p})^{i}\langle\mathfrak{p}\rangle_{\nu}^{j}=\omega(\mathfrak{p})^{j}\langle\mathfrak{p}\rangle_{\nu}^{j}=\iota\left(\mathfrak{p}^{s_{1}}\right)^{j}=\mathfrak{p}^{s_{j}}=\pi_{*}^{-j d_{\mathfrak{p}}}\langle\mathfrak{p}\rangle_{\infty}^{j}=\Psi_{j}\left(\phi_{\mathfrak{p}}^{-1}\right) \pi_{*}^{-j d_{\mathfrak{p}}} .
$$

Therefore

$$
\begin{aligned}
\Psi_{j, i}\left(\Theta_{S}(X)\right) & =\prod_{\mathfrak{p} \notin S}\left(1-\Psi_{j, i}\left(\phi_{\mathfrak{p}}^{-1}\right) X^{d_{\mathfrak{p}}}\right)^{-1} \\
& =\prod_{\mathfrak{p} \notin S}\left(1-\Psi_{j}\left(\phi_{\mathfrak{p}}^{-1}\right) \pi_{\star}^{-j d_{\mathfrak{p}}} X^{d_{\mathfrak{p}}}\right)^{-1}=\Psi_{j}\left(\Theta_{S}\left(\pi_{*}^{-j} X\right)\right),
\end{aligned}
$$

and

$$
L_{\nu}\left(X, j, \omega^{i}\right)=\Psi_{j}\left(\Theta_{S}\left(\pi_{*}^{-j} X\right)\right) \cdot \prod_{\substack{\mathfrak{p} \in S \\ \mathfrak{p} \neq \infty, \nu}}\left(1-\mathfrak{p}^{s_{j}} X^{d_{\mathfrak{p}}}\right)^{-1}
$$


The same arguments used to obtain $(6)$, yield $Z(X, j)=\prod_{\mathfrak{p} \neq \infty}\left(1-\langle\mathfrak{p}\rangle_{\infty}^{j}\left(\pi_{*}^{-j} X\right)^{d_{\mathfrak{p}}}\right)^{-1}$ which can be rewritten as

$$
Z(X, j)=\Psi_{j}\left(\Theta_{S}\left(\pi_{\star}^{-j} X\right)\right) \cdot \prod_{\substack{\mathfrak{p} \in S \\ \mathfrak{p} \neq \infty}}\left(1-\mathfrak{p}^{s_{j}} X^{d_{\mathfrak{p}}}\right)^{-1} .
$$

Together with (7), this leads to $L_{\nu}\left(X, j, \omega^{i}\right)=Z(X, j)\left(1-\nu^{s_{j}} X^{d_{\nu}}\right)$.

(b) For every ideal $\mathfrak{a}$ we have $\langle\mathfrak{a}\rangle_{\nu}^{y} \equiv 1 \equiv\langle\mathfrak{a}\rangle_{\nu}^{i}(\bmod \bar{\nu})$. Hence

$$
\begin{aligned}
L_{\nu}\left(X, y, \omega^{i}\right) & =\sum_{n \geqslant 0}\left(\sum_{\substack{\mathfrak{a} \in \mathcal{I}_{\nu}, \mathfrak{a} \geqslant 0 \\
\operatorname{deg}(\mathfrak{a})=n}} \omega(\mathfrak{a})^{i}\langle\mathfrak{a}\rangle_{\nu}^{y}\right) X^{n} \\
& \equiv \sum_{n \geqslant 0}\left(\sum_{\substack{\mathfrak{a} \in \mathcal{I}_{\nu}, \mathfrak{a} \geqslant 0 \\
\operatorname{deg}(\mathfrak{a})=n}} \omega(\mathfrak{a})^{i}\langle\mathfrak{a}\rangle_{\nu}^{i}\right) X^{n} \quad(\bmod \bar{\nu})=L_{\nu}\left(X, i, \omega^{i}\right) \quad(\bmod \bar{\nu}) \\
& =Z(X, i)\left(1-\nu^{s_{i}} X^{d_{\nu}}\right) \quad(\bmod \bar{\nu}) \equiv Z(X, i) \quad(\bmod \bar{\nu}) .
\end{aligned}
$$

\section{Stickelberger SERIES AND Class Groups}

In this section we shall deal with the algebraic side of the theory: we study the $p$-part of class groups of degree zero divisors of the finite subextension of a $\mathbb{Z}_{p}^{\infty}$-extension of $F$ generated by the torsion of a Hayes module, the main result is the computation of the Fitting ideal of the inverse limit of such groups (Theorem 5.22), which turns out to be generated by (a specialization of) our Stickelberger series. This provides one instance of the Main Conjecture in our setting: the link between the Fitting ideal (i.e. the Stickelberger series) and the various $L$-functions has been described in details in the previous sections. Our strategy, which heavily relies on the computations of Greither and Popescu in [17] and [18], puts emphasis on finite subextensions: this approach, closer to the classical one in characteristic zero, has been suggested and developed in [2] for $F=\mathbb{F}_{q}(t)$.

Remark 5.1. An alternative path using limits of characteristic ideals has been described in [5], where the authors study $\mathbb{Z}_{p}^{\infty}$-extensions of global function fields using $\mathbb{Z}_{p}^{d}$-filtrations (and the Main Conjecture for them proved originally by Crew in [13] and more recently by Burns and Khue, Lai and Tan in [9]). This approach has been applied also to Iwasawa theory for elliptic curves and abelian varieties in [6, building on structure theorems for Selmer groups (see e.g. [29], 30] and [7]) and the only avaliable Main Conjecture in this setting, i.e. the one for constant abelian varieties in [21. Applications to a Main Conjecture for $\mathbb{Z}_{p}^{\infty}$-extensions and to Akashi series are provided in [6. Theorem 3.10] and [8. Section 3]. It would be interesting to try to apply the finite subextension approach to this abelian varieties setting as well.

Some results of this section (basically Theorems 5.10 and 5.17) already appeared in 4 , Section 3] as a specialization of more general computations provided in [4, Section 2]. We decided to include a short account here as well for completeness.

Throughout this chapter we will assume that $d_{\infty}=1$ and that $p$ does not divide $h^{0}(F)$. Under these assumptions we have the following simplifications:

- the residue field $\mathbb{F}_{\infty}$ coincides with the field of constants $\mathbb{F}_{q}$;

- every principal ideal admits a positive generator; 
- the class number of the ring of integers $A$ is equal to $h^{0}(F)$;

- the field $F_{\infty, \mathbf{V}}$ coincides with the field $F_{\infty}$;

- for every $a \in F: \operatorname{deg}(a)=-v_{\infty}(a)$;

- $\pi_{*}=\pi_{\infty}$.

As mentioned in the introduction the first assumption is needed to ensure that all extensions we deal with are geometric and it is not really restrictive because we can reduce to this case by extending the constant field of $F$. The assumption on $h^{0}(F)$ is more crucial (but not too restrictive) because we are going to deal with the characters of a Galois group whose cardinality is divisible by $h^{0}(F)$ (see Section 5.2.2).

5.1. Hayes extensions. Let $H_{A}$ be the Hilbert class field of $A$, i.e. the maximal abelian unramified extension of $F$ which is totally split at $\infty$. Since the prime $\infty$ has degree 1 , the constant field of $H_{A}$ is $\mathbb{F}_{q}$. Class field theory implies that $\operatorname{Pic}(A) \simeq \operatorname{Gal}\left(H_{A} / F\right)$ and the isomorphism is provided by the Artin reciprocity map: in particular the class of a fractional ideal $\mathfrak{a}$ is sent to its Frobenius in $\operatorname{Gal}\left(H_{A} / F\right)$ and, in case the support of $\mathfrak{a}$ is disjoint from $S$, this is simply the restriction of its Artin symbol $\phi_{\mathfrak{a}} \in G_{S}$.

Definition 5.2. We denote by $H_{A}\{\tau\}$ the ring of skew-polynomials in the variable $\tau$ with coefficients in $H_{A}$, where $\tau f=f^{q} \tau$. A Hayes module (or sign-normalized rank 1 Drinfeld module) is a homomorphism of $\mathbb{F}_{q}$-algebras $\Phi: A \rightarrow H_{A}\{\tau\}$, such that:

(a) the image of $A$ is not contained in $H_{A}$;

(b) for every $a \in A$ the coefficient of degree 0 of $\Phi_{a}:=\Phi(a)$ is equal to a;

(c) for every $a \in A$ the degree in $\tau$ of $\Phi_{a}$ is equal to $\operatorname{deg}(a)$ (i.e. $\Phi$ has rank 1);

(d) for every $a \in A$, the leading coefficient of $\Phi_{a}$ is $\operatorname{sgn}(a)$ (i.e. $\Phi$ is sign-normalized).

For details on the Hayes modules and on the properties mentioned below, the reader may refer to [14, Chapter 7], [19] and [26].

For every $a \in A$ and $x \in \bar{F}$ put $a \cdot x:=\Phi_{a}(x)$; this defines an $A$-module structure on $\bar{F}$. For any integral ideal $\mathfrak{a}$ of $A$, consider the left ideal of $H_{A}\{\tau\}$ generated by all the elements $\Phi_{a}$ with $a \in \mathfrak{a}$; since $H_{A}\{\tau\}$ is right-euclidean, we have that every left ideal is principal and we denote by $\Phi_{\mathfrak{a}}$ the unique monic generator of the ideal $\left(\Phi_{a}: a \in \mathfrak{a}\right)$.

Definition 5.3. The a-torsion of $\bar{F}$ is the set

$$
\Phi[\mathfrak{a}]:=\left\{x \in \bar{F}: \Phi_{\mathfrak{a}}(x)=0\right\} .
$$

It is finite for any $\mathfrak{a} \neq 0$ and it is an $A / \mathfrak{a}$-module isomorphic to $A / \mathfrak{a}$.

We put $F(\mathfrak{a}):=H_{A}(\Phi[\mathfrak{a}])$; the following theorem explains how the a-torsion of a Hayes module can be used to define extensions of $F$ analogous to the cyclotomic extension of $\mathbb{Q}$.

Theorem 5.4. The field $F(\mathfrak{a})$ is a geometric, abelian Galois extension of $F$ which verifies

(a) the only ramified primes in $F(\mathfrak{a}) / H_{A}$ are the primes of $H_{A}$ dividing $\mathfrak{a}$ and $\infty$;

(b) $\operatorname{Gal}\left(F(\mathfrak{a}) / H_{A}\right) \simeq(A / \mathfrak{a})^{\times}$via an isomorphism sending $a \in A$ to $\sigma_{a}$, where $\sigma_{a}(\lambda)=$ $\Phi_{a}(\lambda)$ for every $\lambda \in \Phi[\mathfrak{a}]$;

(c) the isomorphism in (b) sends the decomposition and inertia groups of $\infty$ to $\mathbb{F}_{q}^{\times}$;

(d) if $\mathfrak{p}^{n}$ is the exact power of $\mathfrak{p}$ dividing $\mathfrak{a}$, then the isomorphism in (b) sends the inertia group of $\mathfrak{p}$ to $\left(A / \mathfrak{p}^{n}\right)^{\times}$. 
We fix a prime $\mathfrak{p}$ and put $S=\{\mathfrak{p}, \infty\}$. For any $n \geqslant 0$ let $F_{n}:=F\left(\mathfrak{p}^{n+1}\right)$ and $G_{n}=\operatorname{Gal}\left(F_{n} / F\right)$. From part (b) of Theorem 5.4 we have that $F_{n} / F_{0}$ is a finite $p$-extension and that $F_{0} / F$ has degree $h^{0}(F)\left(q^{d_{\mathfrak{p}}}-1\right)$. Since we assumed $h^{0}(F)$ coprime with $p$, we obtain a decomposition $G_{n} \simeq G_{0} \times \Gamma_{n}$, where $\Gamma_{n}=\operatorname{Gal}\left(F_{n} / F_{0}\right)$ is a $p$-group and $G_{0}$ has order prime with $p$. The fields $F_{n}$ form an Iwasawa tower: if we put $\mathcal{F}=\cup F_{n}$, then

$$
G_{\infty}:=\operatorname{Gal}(\mathcal{F} / F)=\lim _{\leftarrow} G_{n} \simeq G_{0} \times \lim _{\leftarrow} \Gamma_{n}=: G_{0} \times \Gamma_{\infty},
$$

with $\Gamma_{\infty} \simeq \mathbb{Z}_{p}^{\infty}$. Note that the only primes which ramify in $\mathcal{F} / F$ are $\mathfrak{p}$ and $\infty$, so $\mathcal{F} \subseteq F_{S}$. The following diagram gives a recap of the fields and Galois groups introduced above.

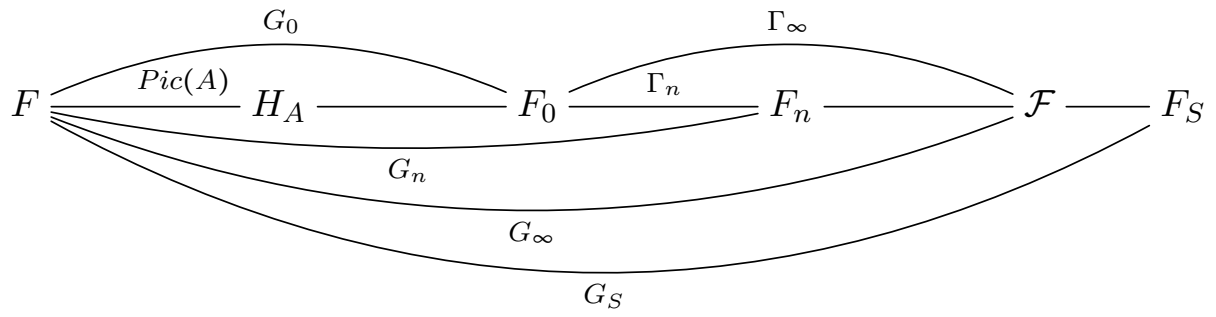

Regarding the behaviour of primes in these extensions, we recall that

- any prime different from $\mathfrak{p}$ and $\infty$ is unramified everywhere;

- $\mathfrak{p}$ is unramified in $H_{A} / F$ and totally ramified in $\mathcal{F} / H_{A}$;

- $\infty$ is totally split in $H_{A} / F$, it ramifies (not totally in general) in $F_{0} / H_{A}$ with inertia group isomorphic to $\mathbb{F}_{q}^{\times}$, and it is again totally split in $\mathcal{F} / F_{0}$.

5.2. Fitting ideals and the Greither-Popescu theorem. Fix an algebraic closure $\overline{\mathbb{F}}_{q}$ of $\mathbb{F}_{q}$ and let $\gamma$ be the arithmetic Frobenius, i.e. a topological generator of $G_{\mathbb{F}}:=\operatorname{Gal}\left(\overline{\mathbb{F}}_{q} / \mathbb{F}_{q}\right)$. For every field $L$ we put $L^{a r}:=\overline{\mathbb{F}}_{q} L$; when $L=F_{n}\left(\right.$ resp. $\mathcal{F}$ ), we have that $F_{n}^{a r}$ (resp. $\mathcal{F}^{a r}$ ) is Galois over $F$, with Galois group isomorphic to $G_{n} \times G_{\mathbb{F}}$ (resp. $G_{\infty} \times G_{\mathbb{F}}$ ) since $F_{n}$ (resp. $\mathcal{F})$ is a geometric extension of $F$.

We recall here the definition of Fitting ideal of a finitely generated module. For an in-depth discussion the reader may refer to [24, Chapter 3] or to the appendix of [22] for the main properties. Let $R$ be any commutative and unitary ring, let $M$ be a finitely generated $R$-module and fix a set of generators $\left\{m_{1}, \ldots, m_{r}\right\}$ for $M$. A relation vector between the generators $m_{i}$ is an element $\underline{a}=\left(a_{1}, \ldots, a_{r}\right) \in R^{r}$ such that $\sum a_{i} m_{i}=0$. A matrix of relations is any $q \times r$ matrix, with $q \geqslant r$, whose rows are relation vectors.

Definition 5.5. The Fitting ideal of $M$, denoted $\operatorname{Fitt}_{R}(M)$, is the ideal generated by the determinants of all the $r \times r$ minors of all the matrices of relations of $M$.

It is well known that $\operatorname{Fitt}_{R}(M)$ does not depend on the chosen set of generators, moreover it is enough to consider square relation matrices of dimension $r$. We recall that $\operatorname{Fitt}_{R}(M) \subseteq$ $\operatorname{Ann}_{R}(M)$, i.e. we can have a nontrivial ideal only for torsion modules.

5.2.1. The modules $H_{n}(\nu)$. For every prime $\nu$ of $F$ there exists only a finite number of primes of $F_{n}^{a r}$ lying above $\nu$, in particular

- there are $d_{\mathfrak{p}} h^{0}(F)$ primes dividing $\mathfrak{p}$;

- there are $h^{0}(F) q^{n d_{\mathfrak{p}}}\left(q^{d_{\mathfrak{p}}}-1\right) /(q-1)$ primes dividing $\infty$. 
Let $H_{n}(\nu)$ be the free $\mathbb{Z}_{p}$-module generated by the primes of $F_{n}^{a r}$ lying above $\nu$. Let $I_{n}(\nu) \subseteq G_{n}$ be the inertia group of $\nu$, since for $\nu=\infty$ such group does not depend on $n$, we shall simply write $I_{n}(\infty)=: I_{\infty}$. We have that $H_{n}(\nu)$ is also a free $\mathbb{Z}_{p}\left[G_{n} / I_{n}(\nu)\right]$-module of rank $d_{\nu}$, and there is a natural action of $G_{\mathbb{F}}$ on $H_{n}(\nu)$. We are interested in studying the structure of $H_{n}(\nu)$ as a $\mathbb{Z}_{p}\left[G_{n}\right] \llbracket G_{\mathbb{F}} \rrbracket$-module.

For $\nu \in\{\mathfrak{p}, \infty\}$ we denote by $\operatorname{Fr}_{\nu}$ any lift to $G_{n}$ of the Frobenius map in $G_{n} / I_{n}(\nu)$. Since the decomposition and inertia groups of $\infty$ coincide, we can choose $\operatorname{Fr}_{\infty}=1$. The same choice is valid for $\mathfrak{p}$ if and only if $\mathfrak{p}$ is totally split in $H_{A}$.

Definition 5.6. The Euler factor at $\nu$ is

$$
e_{\nu}(X):=1-\operatorname{Fr}_{\nu}^{-1} X^{d_{\nu}} \in \mathbb{Z}_{p}\left[G_{n}\right] \llbracket X \rrbracket .
$$

Since we will also need to specialize the variable $X$ at $\gamma^{-1}$, we put

$$
e_{\nu}:=e_{\nu}\left(\gamma^{-1}\right)=1-\operatorname{Fr}_{\nu}^{-1} \gamma^{-d_{\nu}} \in \mathbb{Z}_{p}\left[G_{n}\right] \llbracket G_{\mathbb{F}} \rrbracket .
$$

The statements of [18, Lemmas 2.1 and 2.2], adapted to our setting, translate into

Lemma 5.7. For $\nu \in\{\mathfrak{p}, \infty\}$, let $A u g_{\nu, n}:=\left(\tau-1: \tau \in I_{n}(\nu)\right)$ be the augumentation ideal of $I_{n}(\nu)$ in $\mathbb{Z}_{p}\left[G_{n}\right] \llbracket G_{\mathbb{F}} \rrbracket$. Then

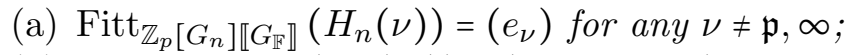
(b) $\operatorname{Fitt}_{\mathbb{Z}_{p}\left[G_{n}\right] \llbracket G_{\mathbb{F}} \rrbracket}\left(H_{n}(\infty)\right)=\left(e_{\infty}, A u g_{\infty, n}\right)$;
(c) $\operatorname{Fitt}_{\mathbb{Z}_{p}\left[G_{n}\right] \llbracket G_{\mathbb{F}} \rrbracket}\left(H_{n}(\mathfrak{p})\right)=\left(e_{\mathfrak{p}}, A_{u g_{\mathfrak{p}, n}}\right)$.

Since the $H_{n}(\nu)$ are free $\mathbb{Z}_{p}\left[G_{n} / I_{n}(\nu)\right]$-modules, we have isomorphisms

- if $\nu \neq \mathfrak{p}, \infty$

$$
H_{n}(\nu) \simeq \mathbb{Z}_{p}\left[G_{n}\right] \llbracket G_{\mathbb{F}} \rrbracket /\left(e_{\nu}\right)
$$

- if $\nu=\infty$

$$
H_{n}(\infty) \simeq \mathbb{Z}_{p}\left[G_{0} / \mathbb{F}_{q}^{\times} \times \Gamma_{n}\right] \llbracket G_{\mathbb{F}} \rrbracket /\left(e_{\infty}\right)
$$

- if $\nu=\mathfrak{p}$

$$
H_{n}(\mathfrak{p}) \simeq \mathbb{Z}_{p}[\operatorname{Pic}(A)] \llbracket G_{\mathbb{F}} \rrbracket /\left(e_{\mathfrak{p}}\right) .
$$

5.2.2. Complex characters. Let $\chi \in \widehat{G_{0}}:=\operatorname{Hom}\left(G_{0}, \mathbb{C}^{\times}\right)$be a complex character for $G_{0}$, to include its values we shall extend scalars to the Witt ring $W=\mathbb{Z}_{p}[\zeta]$, where $\zeta$ denotes any primitive root of unity of order $\left|G_{0}\right|$ (recall that we are assuming $\left(\left|G_{0}\right|, p\right)=1$ ). We let

$$
e_{\chi}:=\frac{1}{\left|G_{0}\right|} \sum_{g \in G_{0}} \chi\left(g^{-1}\right) g \in W\left[G_{0}\right]
$$

be the idempotent associated to $\chi$. For any $W\left[G_{0}\right]$-module $M$, we denote its $\chi$-part by $M(\chi):=e_{\chi} M$. For any $\mathbb{Z}_{p}\left[G_{0}\right]$-module $M$ we shall abuse notations a bit and write $M(\chi)$ to denote $\left(W \otimes_{\mathbb{Z}_{p}} M\right)(\chi)$. This will not have any effect on the numerous exact sequences we are going to consider, because $W$ is a flat $\mathbb{Z}_{p}$-module. Finally recall that if $G_{0}$ acts trivially on $M$, then

$$
M(\chi)= \begin{cases}W \otimes_{\mathbb{Z}_{p}} M & \text { if } \chi=\chi_{0}, \\ 0 & \text { if } \chi \neq \chi_{0}\end{cases}
$$

(as usual $\chi_{0}$ denotes the trivial character).

Definition 5.8. Let $\chi$ be a character of $G_{0}$. We will distinguish 3 types of characters: 
- $\chi$ is said to be of type 1 if $\chi\left(I_{\infty}\right) \neq 1$;

- $\chi$ is said to be of type 2 if $\chi\left(I_{\infty}\right)=1$ and $\chi\left(\operatorname{Gal}\left(F_{0} / H_{A}\right)\right) \neq 1$;

- $\chi$ is said to be of type 3 if $\chi\left(\operatorname{Gal}\left(F_{0} / H_{A}\right)\right)=1$.

Recall that $d_{\infty}=1$ and $\operatorname{Fr}_{\infty}=1$, so that $e_{\infty}=1-\gamma^{-1}$. Taking $\chi$-parts in Lemma 5.7 we get

$$
H_{n}(\infty)(\chi) \simeq \begin{cases}0 & \text { if } \chi \text { is of type } 1, \\ W\left[\Gamma_{n}\right] & \text { otherwise } .\end{cases}
$$

For the prime $\mathfrak{p}$ we have the exact sequence (again from Lemma 5.7)

$$
e_{\chi}\left(1-\mathrm{Fr}_{\mathfrak{p}}^{-1} \gamma^{-d_{\mathfrak{p}}}\right) W[\operatorname{Pic}(A)] \llbracket G_{\mathbb{F}} \rrbracket \hookrightarrow e_{\chi} W[\operatorname{Pic}(A)] \llbracket G_{\mathbb{F}} \rrbracket \longrightarrow H_{n}(\mathfrak{p})(\chi),
$$

which leads to

$$
H_{n}(\mathfrak{p})(\chi) \simeq \begin{cases}0 & \text { if } \chi \text { is of type } 1 \text { or } 2, \\ W \llbracket G_{\mathbb{F}} \rrbracket /\left(1-\chi\left(\mathrm{Fr}_{\mathfrak{p}}^{-1}\right) \gamma^{\left.-d_{\mathfrak{p}}\right)}\right. & \text { otherwise } .\end{cases}
$$

5.2.3. The theorem of Greither and Popescu. Let $X_{n}$ be the projective curve defined over $\mathbb{F}_{q}$ and associated with $F_{n}$; let

$$
\operatorname{Jac}\left(X_{n}\right)\left(\overline{\mathbb{F}}_{q}\right) \simeq \operatorname{Div}^{0}\left(F_{n}^{a r}\right) /\left\{\operatorname{Div}(x): x \in\left(F_{n}^{a r}\right)^{\times}\right\}
$$

be the set of $\overline{\mathbb{F}}_{q}$-rational points of its Jacobian. Following [17, Section 2], we define the Deligne's Picard 1-motive $\mathcal{M}_{n}:=\mathcal{M}_{S}\left(F_{n}^{a r}\right)$ as the group morphism $\operatorname{Div}^{0}\left(F_{n}^{a r}\right) \rightarrow$ $\operatorname{Jac}\left(X_{n}\right)\left(\overline{\mathbb{F}}_{q}\right)$ which induces the isomorphism above. In terms of [17, Definition 2.3] it is the morphism associated to $\left(X_{n}, \overline{\mathbb{F}}_{q}, S\left(F_{n}^{a r}\right), \varnothing\right)$, where $S\left(F_{n}^{a r}\right)$ is the set of primes of $F_{n}^{a r}$ lying above primes in $S$ and the choice of $\varnothing$ is justified by [17, Remark 2.7]. The $m$-torsion $\mathcal{M}_{n}[m]$ of $\mathcal{M}_{n}$ (see [17, Definition 2.5]) fits into the exact sequence

$$
0 \longrightarrow \operatorname{Jac}\left(X_{n}\right)\left(\overline{\mathbb{F}}_{q}\right)[m] \longrightarrow \mathcal{M}_{n}[m] \longrightarrow \operatorname{Div}^{0}\left(S\left(F_{n}^{a r}\right)\right) \otimes \mathbb{Z} / m \mathbb{Z} \longrightarrow 0
$$

(where $\operatorname{Div}^{0}\left(S\left(F_{n}^{a r}\right)\right.$ ) are the divisors supported on $S\left(F_{n}^{a r}\right)$ ) and behaves well with respect to norm maps so that we can define its $p$-adic Tate module as $T_{p}\left(\mathcal{M}_{n}\right)=\lim _{\leftarrow} \mathcal{M}_{n}\left[p^{m}\right]$.

We denote by $T_{p}\left(F_{n}\right):=T_{p}\left(\operatorname{Jac}\left(X_{n}\right)\left(\overline{\mathbb{F}}_{q}\right)\right)$ the $p$-adic Tate module of the Jacobian of $X_{n}$. Our next task is to study the structure of $T_{p}\left(F_{n}\right)$ as a Galois module over $\mathbb{Z}_{p}\left[\Gamma_{n}\right] \llbracket G_{\mathbb{F}} \rrbracket$ and a crucial role will be played by Theorem 5.9 below.

Let $\Theta_{n}(X)$ (resp. $\left.\Theta_{\infty}(X)\right)$ be the projection of the Stickelberger series $\Theta_{S}(X)$ to the ring $\mathbb{Z}\left[G_{n}\right] \llbracket X \rrbracket$ (resp. $\mathbb{Z} \llbracket G_{\infty} \rrbracket \llbracket X \rrbracket$ ), which is easily seen to be the Stickelberger series associated to the extension $F_{n} / F$ (resp. $\mathcal{F} / F$ ): actually one obtains an equivalent definition for $\Theta_{S}(X)$ by taking the inverse limit (with respect to projections) of the Stickelberger series of the subextensions of $F_{S}$.

In [17, Theorem 4.3] the authors prove the following

Theorem 5.9. One has $\operatorname{Fitt}_{\mathbb{Z}_{p}\left[G_{n}\right] \llbracket G_{\mathbb{E}} \rrbracket}\left(T_{p}\left(\mathcal{M}_{n}\right)\right)=\left(\Theta_{n}\left(\gamma^{-1}\right)\right)$.

Note that evaluating the Stickelberger series $\Theta_{n}(X)$ at $X=\gamma^{-1}$ makes sense because of Proposition 2.4 . 
5.3. Fitting ideals for Tate modules: finite level. We define $D_{n}$ to be the kernel of the deg map in the following exact sequence

$$
0 \longrightarrow D_{n} \longrightarrow H_{n}(\infty) \oplus H_{n}(\mathfrak{p}) \stackrel{\text { deg }}{\longrightarrow} \mathbb{Z}_{p} \longrightarrow 0 .
$$

Since $G_{0}$ acts trivially on $\mathbb{Z}_{p}$ we have

$$
D_{n}(\chi) \simeq \begin{cases}0 & \text { if } \chi \text { is of type } 1, \\ W\left[\Gamma_{n}\right] & \text { if } \chi \text { is of type } 2, \\ W\left[\Gamma_{n}\right] \oplus W \llbracket G_{\mathbb{F}} \rrbracket /\left(1-\chi\left(\mathrm{Fr}_{\mathfrak{p}}^{-1}\right) \gamma^{\left.-d_{\mathfrak{p}}\right)}\right. & \text { if } \chi \text { is of type } 3 \\ & \text { and } \chi \neq \chi_{0} .\end{cases}
$$

In [17, after Definition 2.6] the authors provide the following exact sequence

$$
0 \longrightarrow T_{p}\left(F_{n}\right) \longrightarrow T_{p}\left(\mathcal{M}_{n}\right) \longrightarrow D_{n} \longrightarrow 0 .
$$

For every character $\chi$ we denote by $\Theta_{n}(X, \chi)$ the only element of $\mathbb{Z}\left[\Gamma_{n}\right] \llbracket X \rrbracket$ that satisfies $\Theta_{n}(X, \chi) e_{\chi}=e_{\chi} \Theta_{n}(X)$. Then we have the following (see also [4, Theorem 3.2])

Theorem 5.10. Let $\chi \in \widehat{G_{0}}$ be a character of type 1 or 2 . Then

$$
\operatorname{Fitt}_{W\left[\Gamma_{n}\right] \llbracket G_{\mathbb{F}} \rrbracket}\left(T_{p}\left(F_{n}\right)(\chi)\right)=\left(\Theta_{n}^{\sharp}\left(\gamma^{-1}, \chi\right)\right),
$$

where we put

$$
\Theta_{n}^{\sharp}\left(\gamma^{-1}, \chi\right)= \begin{cases}\Theta_{n}\left(\gamma^{-1}, \chi\right) & \text { if } \chi \text { is of type } 1, \\ \frac{\Theta_{n}\left(\gamma^{-1}, \chi\right)}{1-\gamma^{-1}} & \text { if } \chi \text { is of type } 2 .\end{cases}
$$

Proof. Take $\chi$-parts in $(12)$ and use (11) to get:

- if $\chi$ is of type $1, D_{n}(\chi)=0$ and $T_{p}\left(F_{n}\right)(\chi) \simeq T_{p}\left(\mathcal{M}_{n}\right)(\chi)$;

- if $\chi$ is of type $2, D_{n}(\chi)=W\left[\Gamma_{n}\right] \simeq W\left[\Gamma_{n}\right] \llbracket G_{\mathbb{F}} \rrbracket /\left(1-\gamma^{-1}\right)$ is a cyclic $W\left[\Gamma_{n}\right] \llbracket G_{\mathbb{F}} \rrbracket-$ module: hence we can apply [11, Lemma 3] and obtain

$$
\left(1-\gamma^{-1}\right) \operatorname{Fitt}_{W\left[\Gamma_{n}\right] \llbracket G_{\mathbb{F}} \rrbracket}\left(T_{p}\left(F_{n}\right)(\chi)\right)=\operatorname{Fitt}_{W\left[\Gamma_{n}\right] \llbracket G_{\mathbb{F}} \rrbracket}\left(T_{p}\left(\mathcal{M}_{n}\right)(\chi)\right)=\left(\Theta_{n}\left(\gamma^{-1}, \chi\right)\right) \text {. }
$$

When $\chi$ is a character of type 3 things get more involved, since $D_{n}(\chi)$ is not cyclic and we can only compute the Fitting ideal of some dual of the Tate module: details can be found in [12, Section 2.5] or in [4, Section 3].

5.4. Fitting ideals for Tate modules: infinite level. To prove an analog of Theorem 5.10 for the infinite level $\mathcal{F}^{\text {ar }}$ we need to study the relation between $T_{p}\left(F_{n}\right)$ and $T_{p}\left(F_{m}\right)$ for $n \geqslant m$. Let $\Gamma_{m}^{n}:=\operatorname{Gal}\left(F_{n} / F_{m}\right)$ and $I_{\Gamma_{m}^{n}}$ the associated augmentation ideal in $W\left[\Gamma_{n}\right]$. We recall that $F_{n} / F_{m}$ is totally ramified at all primes lying above $\mathfrak{p}$ and unramified everywhere else, moreover the number of primes in $F_{m}$ above $\mathfrak{p}$ is the same for all $m$ and coincides with the number of primes of $H_{A}$ lying above $\mathfrak{p}$.

We denote by $\bar{C}_{n}$ the $p$-part of the class group of degree zero divisors of $F_{n}^{a r}$ and we recall that $T_{p}\left(F_{n}\right)=\operatorname{Hom}\left(\mathbb{Q}_{p} / \mathbb{Z}_{p}, \bar{C}_{n}\right)$. Thus there are natural maps induced by norms and inclusions respectively $N_{m}^{n}: T_{p}\left(F_{n}\right) \rightarrow T_{p}\left(F_{m}\right)$ and $i_{n}^{m}: T_{p}\left(F_{m}\right) \rightarrow T_{p}\left(F_{n}\right)$. We define

$$
T_{p}(\mathcal{F})(\chi)=\lim _{20} T_{p}\left(F_{n}\right)(\chi),
$$


where the limit is taken with respect to the norm maps. The limit $T_{p}(\mathcal{F})(\chi)$ is a module over the profinite (non-noetherian) algebra $\Lambda_{\mathbb{F}}:=W \llbracket \Gamma_{\infty} \rrbracket \llbracket G_{\mathbb{F}} \rrbracket$; our next goal is to compute its Fitting ideal.

Norms and inclusions are defined on the modules $T_{p}\left(\mathcal{M}_{n}\right)$ and $D_{n}$ as well: in particular $i_{n}^{m}: T_{p}\left(\mathcal{M}_{m}\right) \hookrightarrow T_{p}\left(\mathcal{M}_{n}\right)$ is injective and satisfies $T_{p}\left(\mathcal{M}_{n}\right)^{\Gamma_{m}^{n}}=i_{n}^{m}\left(T_{p}\left(\mathcal{M}_{m}\right)\right.$ ) (see [17, Theorem 3.1]) and the composition $N_{m}^{n} \circ i_{n}^{m}$ is multiplication by $\left[F_{n}: F_{m}\right]$ (on both modules). All these maps are compatible with the exact sequence (12), i.e. the following diagram is commutative for every pair of indices $n>m$

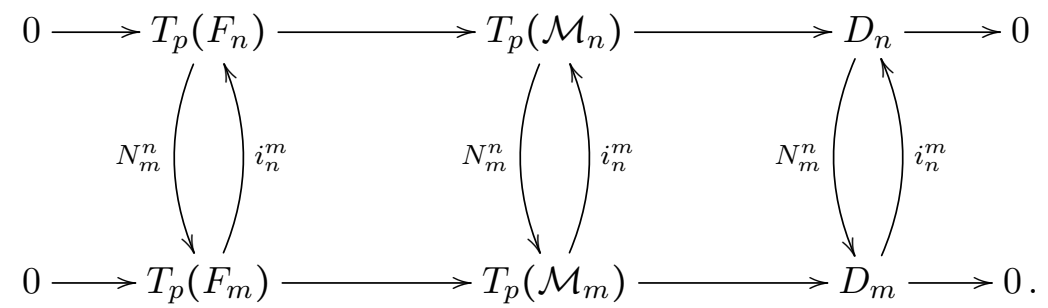

Lemma 5.11. The map $N_{m}^{n}: T_{p}\left(\mathcal{M}_{n}\right) \rightarrow T_{p}\left(\mathcal{M}_{m}\right)$ is surjective, its kernel is $I_{\Gamma_{m}^{n}} T_{p}\left(\mathcal{M}_{n}\right)$.

Proof. By [17, Theorem 3.9 part (2)] $T_{p}\left(\mathcal{M}_{n}\right)$ is a free $\mathbb{Z}_{p}\left[\Gamma_{m}^{n}\right]$-module (because $\Gamma_{m}^{n}$ is a $p$-group), hence cohomologically trivial, i.e.

$$
\widehat{H}^{i}\left(\Gamma_{m}^{n}, T_{p}\left(\mathcal{M}_{n}\right)\right)=0 \text { for every integer } i,
$$

where the $\widehat{H}^{i}\left(\Gamma_{m}^{n}, \bullet\right)$ are Tate cohomology groups.

Specializing the previous equality at $i=0$ we obtain

$$
N_{m}^{n}\left(T_{p}\left(\mathcal{M}_{n}\right)\right)=T_{p}\left(\mathcal{M}_{n}\right)^{\Gamma_{m}^{n}}=i_{n}^{m}\left(T_{p}\left(\mathcal{M}_{m}\right)\right) \simeq T_{p}\left(\mathcal{M}_{m}\right) .
$$

In a similar way we obtain the second part of the lemma by specializing at $i=-1$.

We can now prove an analogous result for the modules $T_{p}\left(F_{n}\right)$.

Proposition 5.12. Let $\chi$ be a character of type 1 or 2 . Then the norm $N_{m}^{n}: T_{p}\left(F_{n}\right)(\chi) \rightarrow$ $T_{p}\left(F_{m}\right)(\chi)$ is surjective and its kernel is $I_{\Gamma_{m}^{n}} T_{p}\left(F_{n}\right)(\chi)$.

Proof. Consider the $\chi$-part of the exact sequence $(12)$, i.e.

$$
0 \longrightarrow T_{p}\left(F_{n}\right)(\chi) \longrightarrow T_{p}\left(\mathcal{M}_{n}\right)(\chi) \longrightarrow D_{n}(\chi) \longrightarrow 0 .
$$

If $\chi$ is a character of type 1 , then $T_{p}\left(F_{n}\right)(\chi) \simeq T_{p}\left(\mathcal{M}_{n}\right)(\chi)$ and this is a restatement of Lemma 5.11.

If $\chi$ is of type 2 , then $D_{n}(\chi) \simeq W\left[\Gamma_{n}\right]$ so both $D_{n}(\chi)$ and $T_{p}\left(\mathcal{M}_{n}\right)(\chi)$ are cohomologically trivial. Hence

$$
\widehat{H}^{i}\left(\Gamma_{m}^{n}, T_{p}\left(F_{n}\right)(\chi)\right)=0 \text { for every integer } i
$$

and, specializing at $i=-1$, we obtain the statement for the kernel.

To prove surjectivity we take $\Gamma_{m}^{n}$-invariants in 13 to obtain

$$
0 \longrightarrow T_{p}\left(F_{n}\right)(\chi)^{\Gamma_{m}^{n}} \longrightarrow T_{p}\left(\mathcal{M}_{m}\right)(\chi) \longrightarrow D_{n}(\chi)^{\Gamma_{m}^{n}} \longrightarrow \widehat{H}^{1}\left(\Gamma_{m}^{n}, T_{p}\left(F_{n}\right)(\chi)\right) \text {. }
$$


Note that $D_{n}(\chi)^{\Gamma_{m}^{n}} \simeq W\left[\Gamma_{n}\right]_{m}^{\Gamma_{m}^{n}} \simeq W\left[\Gamma_{m}\right] \simeq D_{m}(\chi)$, so, comparing with 13) with $m$ in place of $n$, we get $T_{p}\left(F_{n}\right)(\chi)^{\Gamma_{m}^{n}} \simeq T_{p}\left(F_{m}\right)(\chi)$. Now specializing (14) at $i=0$ we obtain

$$
N_{m}^{n}\left(T_{p}\left(F_{n}\right)(\chi)\right)=T_{p}\left(F_{n}\right)(\chi)^{\Gamma_{m}^{n}} \simeq T_{p}\left(F_{m}\right)(\chi) .
$$

We are now ready to prove the main theorems of this section.

Theorem 5.13. Let $\chi$ be a character of type 1 or 2 . Then $T_{p}(\mathcal{F})(\chi)$ is a finitely generated $\Lambda_{\mathbb{F}}$-module. Moreover, if $\lim _{\leftarrow} \Theta_{n}^{\sharp}\left(\gamma^{-1}, \chi\right) \neq 0$, then $T_{p}(\mathcal{F})(\chi)$ is torsion.

Proof. Fix $m$, and let $\mathfrak{I}_{m}$ be the augumentation ideal of $\operatorname{Gal}\left(\mathcal{F} / F_{m}\right)$ in $\Lambda:=W \llbracket \Gamma_{\infty} \rrbracket$. Let $\widetilde{\mathfrak{I}}_{m}=\Lambda_{\mathbb{F}} \otimes_{W \llbracket \Gamma_{\infty} \rrbracket} \Im_{m}$ be the corresponding ideal in $\Lambda_{\mathbb{F}}=\Lambda \llbracket G_{\mathbb{F}} \rrbracket$, and recall that $\mathfrak{I}_{m}=\underset{\leftarrow}{\lim } I_{\Gamma_{m}^{n}}$. By Proposition 5.12 we have

$$
T_{p}\left(F_{m}\right)(\chi)=N_{m}^{n}\left(T_{p}\left(F_{n}\right)(\chi)\right) \simeq T_{p}\left(F_{n}\right)(\chi) / I_{\Gamma_{m}^{n}} T_{p}\left(F_{n}\right)(\chi) .
$$

This holds for every $n>m$ and so

$$
T_{p}\left(F_{m}\right)(\chi) \simeq T_{p}(\mathcal{F})(\chi) / \widetilde{\mathfrak{I}}_{m} T_{p}(\mathcal{F})(\chi) .
$$

The module on the left is finitely generated over $W\left[\Gamma_{m}\right] \llbracket G_{\mathbb{F}} \rrbracket=\Lambda_{\mathbb{F}} / \widetilde{\mathfrak{I}}_{m}$ and, since the ideals $\widetilde{\mathfrak{I}}_{m}$ form an open filtration of the profinite algebra $\Lambda_{\mathbb{F}}$, we can apply the generalized Nakayama Lemma of [3] and obtain that $T_{p}(\mathcal{F})(\chi)$ is a finitely generated $\Lambda_{\mathbb{F}}$-module.

Now we define the element $\Theta_{\infty}^{\sharp}\left(\gamma^{-1}, \chi\right) \in \Lambda_{\mathbb{F}}$ as

$$
\Theta_{\infty}^{\sharp}\left(\gamma^{-1}, \chi\right)= \begin{cases}\Theta_{\infty}\left(\gamma^{-1}, \chi\right) & \text { if } \chi \text { is of type 1, } \\ \frac{\Theta_{\infty}\left(\gamma^{-1}, \chi\right)}{1-\gamma^{-1}} & \text { if } \chi \text { is of type 2, }\end{cases}
$$

i.e. the inverse limit of the generators of $\operatorname{Fitt}_{W\left[\Gamma_{m}\right] \llbracket G_{\mathbb{F}} \rrbracket}\left(T_{p}\left(F_{m}\right)(\chi)\right)$, by Theorem 5.10 . Clearly $\Theta_{\infty}^{\sharp}\left(\gamma^{-1}, \chi\right) T_{p}(\mathcal{F})(\chi)=0$ and this implies the statement on torsion.

Now we know that the Fitting ideal of $T_{p}(\mathcal{F})(\chi)$ is well defined and we proceed to compute a generator via a limit process.

Theorem 5.14. Let $\chi$ be a character of type 1 or 2 . Then

$$
\operatorname{Fitt}_{\Lambda_{\mathbb{F}}}\left(T_{p}(\mathcal{F})(\chi)\right)=\left(\Theta_{\infty}^{\sharp}\left(\gamma^{-1}, \chi\right)\right) .
$$

Proof. The equalities

$$
\left(\Theta_{\infty}^{\sharp}\left(\gamma^{-1}, \chi\right)\right)=\lim _{\leftarrow}\left(\Theta_{n}^{\sharp}\left(\gamma^{-1}, \chi\right)\right)=\lim _{\leftarrow} \operatorname{Fitt}_{W\left[\Gamma_{n}\right] \llbracket G_{\mathbb{F}} \rrbracket}\left(T_{p}\left(F_{n}\right)(\chi)\right)
$$

reduce the proof to showing

$$
\operatorname{Fitt}_{\Lambda_{\mathbb{F}}}\left(T_{p}(\mathcal{F})(\chi)\right)=\lim _{\leftarrow} \operatorname{Fitt}_{W\left[\Gamma_{n}\right] \llbracket G_{\mathbb{F}} \rrbracket}\left(T_{p}\left(F_{n}\right)(\chi)\right) .
$$

Let $N_{m}^{\infty}: T_{p}(\mathcal{F})(\chi) \rightarrow T_{p}\left(F_{m}\right)(\chi)$ be induced by the projection modulo $\widetilde{\mathfrak{I}}_{m} T_{p}(\mathcal{F})(\chi)$. These maps are obviously compatible with the norm maps, i.e. $N_{m}^{\infty}=N_{m}^{n} \circ N_{n}^{\infty}$ for any $n>m$. Let $t_{1}, \ldots, t_{r}$ be $\Lambda_{\mathbb{F}^{-}}$generators of $T_{p}(\mathcal{F})(\chi)$ and write $K_{\infty}$ for the kernel of the surjective map $\Lambda_{\mathbb{F}}^{\oplus r} \rightarrow T_{p}(\mathcal{F})(\chi)$ sending the $i$-th element of the canonical basis to $t_{i}$. We have an exact sequence

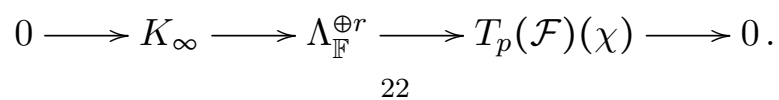


Since $N_{n}^{\infty}\left(t_{1}\right), \ldots, N_{n}^{\infty}\left(t_{r}\right)$ generate $T_{p}\left(F_{n}\right)(\chi)$ over $W\left[\Gamma_{n}\right] \llbracket G_{\mathbb{F}} \rrbracket$, we can construct similar exact sequences for every integer $n$, i.e.

$$
0 \longrightarrow K_{n} \longrightarrow W\left[\Gamma_{n}\right] \llbracket G_{\mathbb{F}} \rrbracket^{\oplus r} \longrightarrow T_{p}\left(F_{n}\right)(\chi) \longrightarrow 0
$$

(where now the $i$-th element of the canonical basis maps to $N_{n}^{\infty}\left(t_{i}\right)$ ). They all fit into the commutative diagrams

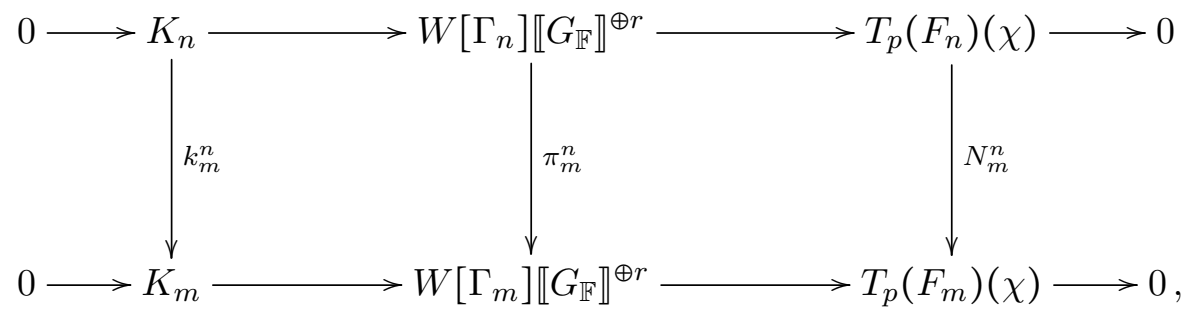

where $k_{m}^{n}:=\left(\pi_{m}^{n}\right)_{\mid K_{n}}$.

One has $\operatorname{Ker}\left(\pi_{m}^{n}\right)=\left(I_{\Gamma_{m}^{n}} W\left[\Gamma_{n}\right] \llbracket G_{\mathbb{F}} \rrbracket\right)^{\oplus r}$ and $\operatorname{Ker}\left(N_{m}^{n}\right)=I_{\Gamma_{m}^{n}} T_{p}\left(F_{n}\right)(\chi)$ (by Proposition 5.12 , so the map between them is surjective. Since $\pi_{m}^{n}$ is surjective, the snake lemma implies that $k_{m}^{n}$ is surjective as well and the diagram above satisfies the Mittag-Leffler condition which allows us to take the inverse limit. Comparing this limit with (15), we obtain $K_{\infty}=\lim K_{n}$.

Let $\mathscr{M}_{r}\left(K_{m}\right)$ be the set of $r \times r$ matrices whose entries are in $W\left[\Gamma_{m}\right] \llbracket G_{\mathbb{F}} \rrbracket$ and such that each row, seen as a vector in $W\left[\Gamma_{m}\right] \llbracket G_{\mathbb{F}} \rrbracket^{\oplus r}$, is in $K_{m}$; we still denote by $k_{m}^{n}$ the natural extension of the map $K_{n} \rightarrow K_{m}$ to $\mathscr{M}_{r}\left(K_{n}\right) \rightarrow \mathscr{M}_{r}\left(K_{m}\right)$, which is surjective as well. By definition $\operatorname{Fitt}_{W\left[\Gamma_{m}\right] \llbracket G_{\mathbb{F}} \rrbracket}\left(T_{p}\left(F_{m}\right)(\chi)\right)$ is the ideal generated by $\left\{\operatorname{det}\left(M_{m}\right): M_{m} \in \mathscr{M}_{r}\left(K_{m}\right)\right\}$. The commutativity of the previous diagram yields for each $M_{n} \in \mathscr{M}_{r}\left(K_{n}\right)$

$$
\pi_{m}^{n}\left(\operatorname{det}\left(M_{n}\right)\right)=\operatorname{det}\left(k_{m}^{n}\left(M_{n}\right)\right) .
$$

Extending this construction to the infinite level (with analogous notations), we obtain $\pi_{m}^{\infty}\left(\operatorname{det}\left(M_{\infty}\right)\right) \in \operatorname{Fitt}_{W\left[\Gamma_{m}\right] \llbracket G_{\mathbb{I}} \rrbracket}\left(T_{p}\left(F_{m}\right)(\chi)\right)$ for any $M_{\infty} \in \mathscr{M}_{r}\left(K_{\infty}\right)$. Hence, for any $m$, $\pi_{m}^{\infty}\left(\operatorname{Fitt}_{\Lambda_{\mathbb{F}}}\left(T_{p}(\mathcal{F})(\chi)\right)\right) \subseteq \operatorname{Fitt}_{W\left[\Gamma_{m}\right] \llbracket G_{\mathbb{F}} \rrbracket}\left(T_{p}\left(F_{m}\right)(\chi)\right)$, and

$$
\operatorname{Fitt}_{\Lambda_{\mathbb{F}}}\left(T_{p}(\mathcal{F})(\chi)\right) \subseteq \lim _{\leftarrow} \operatorname{Fitt}_{W\left[\Gamma_{m}\right] \llbracket G_{\mathbb{F}} \rrbracket}\left(T_{p}\left(F_{m}\right)(\chi)\right) .
$$

The other inclusion needs a little bit more work, basically we follow the arguments of [16, Theorem 2.1]. Each element of $\operatorname{Fitt}_{W\left[\Gamma_{m}\right] \llbracket G_{\mathbb{E}} \rrbracket}\left(T_{p}\left(F_{m}\right)(\chi)\right)$ can be written as a linear combination

$$
x_{m}=\sum_{i=1}^{s} \lambda_{i} \operatorname{det}\left(M_{m}^{(i)}\right)
$$

with $\lambda_{i} \in W\left[\Gamma_{m}\right] \llbracket G_{\mathbb{H}} \rrbracket$ and $M_{m}^{(i)} \in \mathscr{M}_{r}\left(K_{m}\right)$; multiplying the first row of the $M_{m}^{(i)}$ by $\lambda_{i}$, we get matrices $\bar{M}_{m}^{(i)}$ such that

$$
x_{m}=\sum_{i=1}^{s} \operatorname{det}\left(\bar{M}_{m}^{(i)}\right),
$$

i.e. we can assume that all coefficients in (16) are 1. Since the number of elements needed to generate $T_{p}\left(F_{m}\right)(\chi)$ (and $T_{p}(\mathcal{F})(\chi)$ ) can be chosen independently from $m$, one has that $s$ can be chosen independently of $m$ as well. 
Now put $\mathcal{B}_{m}:=\mathscr{M}_{r}\left(K_{m}\right)^{\oplus s}$ with the induced topology and define the non-linear operator $\phi_{m}: \mathcal{B}_{m} \rightarrow W\left[\Gamma_{m}\right] \llbracket G_{\mathbb{F}} \rrbracket$, by $\phi_{m}\left(M_{m}^{(1)}, \ldots, M_{m}^{(s)}\right)=\sum_{i} \operatorname{det}\left(M_{m}^{(i)}\right)$ (analogous definition for $\left.\phi_{\infty}\right)$. This operator is continuous and its image is $\operatorname{Fitt}_{W\left[\Gamma_{m}\right] \llbracket G_{\mathbb{F}} \rrbracket} T_{p}\left(F_{m}\right)(\chi)$. We extend the map $k_{m}^{n}$ from $\mathscr{M}_{r}\left(K_{n}\right)$ to $\mathcal{B}_{n}$ and get the commutative diagram

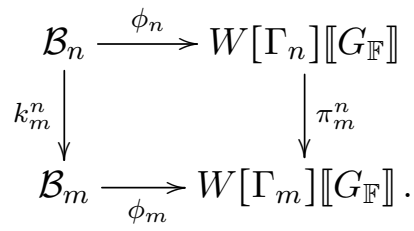

Now we take a sequence $\left(x_{m}\right)_{m \in \mathbb{N}} \in \lim _{\leftarrow} \operatorname{Fitt}_{W\left[\Gamma_{m}\right] \llbracket G_{\mathbb{F}} \rrbracket}\left(T_{p}\left(F_{m}\right)(\chi)\right)$, and look for an element $b_{\infty} \in \mathcal{B}_{\infty}$ such that $\left(x_{m}\right)_{m \in \mathbb{N}}=\phi_{\infty}\left(b_{\infty}\right) \in \operatorname{Fitt}_{\Lambda_{\mathbb{F}}}\left(T_{p}(\mathcal{F})(\chi)\right)$.

For any $m$ put $\Upsilon_{m}:=\phi_{m}^{-1}\left(x_{m}\right)$, then $\Upsilon_{m}$ is closed and, since $W\left[\Gamma_{m}\right] \llbracket G_{\mathbb{F}} \rrbracket$ is compact, $\Upsilon_{m}$ is compact as well. For each $v_{n} \in \Upsilon_{n}$ we have that

$$
\phi_{m}\left(k_{m}^{n}\left(v_{n}\right)\right)=\pi_{m}^{n}\left(\phi_{n}\left(v_{n}\right)\right)=\pi_{m}^{n}\left(x_{n}\right)=x_{m},
$$

thus $k_{m}^{n}\left(\Upsilon_{n}\right) \subseteq \Upsilon_{m}$ and we define

$$
\bar{\Upsilon}_{m}=\bigcap_{n>m} k_{m}^{n}\left(\Upsilon_{n}\right) \subseteq \Upsilon_{m}
$$

Since $k_{m}^{n}$ is continuous, $\bar{\Upsilon}_{m}$ is compact and not empty, moreover one easily shows that $k_{m}^{n}\left(\bar{\Upsilon}_{n}\right) \subseteq \bar{\Upsilon}_{m}$ and we are going to prove equality between them. Let $\bar{v}_{m} \in \bar{\Upsilon}_{m}$ so that, for any $n>m$, there exists $v_{n} \in \Upsilon_{n}$ with $k_{m}^{n}\left(v_{n}\right)=\bar{v}_{m}$. Now fix $\ell>0$ and, for $n>m+\ell$, consider $k_{m+\ell}^{n}\left(v_{n}\right) \in \Upsilon_{m+\ell}$ as a sequence in $n$. Since $\Upsilon_{m+\ell}$ is compact, there exists a convergent subsequence $v_{n_{j}}$ whose limit we call $\bar{v}_{m+\ell}$. Then, for any $n>m+\ell$,

$$
\bar{v}_{m+\ell}=\lim _{j \rightarrow \infty} k_{m+\ell}^{n_{j}}\left(v_{n_{j}}\right)=\lim _{\substack{j \rightarrow \infty \\ n_{j} \geqslant n}}\left(k_{m+\ell}^{n} \circ k_{n}^{n_{j}}\right)\left(v_{n_{j}}\right) \in k_{m+\ell}^{n}\left(\Upsilon_{n}\right),
$$

i.e. $\bar{v}_{m+\ell}$ is in $\bar{\Upsilon}_{m+\ell}$. Obviously $k_{m}^{m+\ell}\left(\bar{v}_{m+\ell}\right)=\bar{v}_{m}$, so the map $k_{m}^{m+\ell}$ is surjective and we have constructed a coherent sequence $b_{\infty}:=\bar{v}_{\infty}=\left(\bar{v}_{m}\right)_{m \in \mathbb{N}} \in \mathcal{B}_{\infty}$. Since $\phi_{m}\left(\bar{v}_{m}\right)=x_{m}$ for each integer $m$, we have that $\phi_{\infty}\left(b_{\infty}\right)=\left(x_{m}\right)_{m \in \mathbb{N}}$.

5.5. Fitting ideals for the class groups: finite level. Now we move to our primary interest: the $p$-part $\mathcal{C} \ell^{0}\left(F_{n}\right)$ of the class groups of degree zero divisors of the field $F_{n}$ which, from now on, we shall denote by $C_{n}$ and which is naturally a finitely generated torsion $W\left[\Gamma_{n}\right]$-module. To compute its Fitting ideal we shall use specializations of $\operatorname{Fitt}_{W\left[\Gamma_{n}\right] \llbracket G_{\mathbb{F}} \rrbracket}\left(T_{p}\left(F_{n}\right)(\chi)\right)$ as suggested by the following lemmas (for the first see e.g. [2, Lemma 4.6], the second is a well known property of Fitting ideals).

Lemma 5.15. There is an isomorphism of $\mathbb{Z}_{p}\left[G_{n}\right]$-modules

$$
T_{p}\left(F_{n}\right)_{G_{\mathbb{F}}}:=T_{p}\left(F_{n}\right) /\left(1-\gamma^{-1}\right) T_{p}\left(F_{n}\right) \simeq C_{n} .
$$

Lemma 5.16. Let $M$ be a finitely generated torsion module over $R$. Let I be any nontrivial ideal of $R$ and consider the projection $\pi_{I}: R \rightarrow R / I$. Then

$$
\operatorname{Fitt}_{R / I}(M / I M)=\pi_{I}\left(\operatorname{Fitt}_{R}(M)\right) .
$$


Let $\pi: W\left[\Gamma_{n}\right] \llbracket G_{\mathbb{F}} \rrbracket \rightarrow W\left[\Gamma_{n}\right] \simeq W\left[\Gamma_{n}\right] \llbracket G_{\mathbb{F}} \rrbracket / I_{G_{\mathbb{F}}}$ be the canonical projection sending $\gamma$ to 1. Combining Lemmas 5.15 and 5.16, and the computations of Theorem 5.10 we obtain

Theorem 5.17. Let $\chi \in \widehat{G_{0}}$ be a character of type 1 or 2. Then

$$
\operatorname{Fitt}_{W\left[\Gamma_{n}\right]}\left(C_{n}(\chi)\right)=\left(\Theta_{n}^{\sharp}(1, \chi)\right),
$$

where

$$
\Theta_{n}^{\sharp}(1, \chi)= \begin{cases}\Theta_{n}(1, \chi) & \text { if } \chi \text { is of type } 1, \\ \left(\frac{\Theta_{n}\left(\gamma^{-1}, \chi\right)}{1-\gamma^{-1}}\right)_{\mid \gamma=1} & \text { if } \chi \text { is of type } 2 .\end{cases}
$$

Proof. Just specialize Theorem 5.10 to $\gamma=1$ recalling the convergence properties of the Stickelberger series.

5.6. Fitting ideals for the class groups: infinite level and the Main Conjecture. Now we approach the $W \llbracket \Gamma \rrbracket$-module $C_{\infty}:=\lim _{\leftarrow} C_{n}$, where the limit is with respect to the norm maps $N_{m}^{n}: C_{n} \rightarrow C_{m}$. We shall also consider maps $i_{n}^{m}: C_{m} \rightarrow C_{n}$ induced by the embeddings $i_{n}^{m}: \operatorname{Div}\left(F_{m}\right) \rightarrow \operatorname{Div}\left(F_{n}\right)$. We recall that for any $D=\sum_{\nu} n_{\nu} \nu \in \operatorname{Div}\left(F_{m}\right)$, we have $i_{n}^{m}(D):=\sum_{\nu} n_{\nu} \sum_{w \mid \nu} e(w \mid \nu) w$, where $e(w \mid \nu)$ is the ramification index of $w$ over $\nu$. In particular, since $\operatorname{deg}\left(i_{n}^{m}(D)\right)=\left[F_{n}: F_{m}\right] \cdot \operatorname{deg}(D)$, the image of a degree zero divisor still has degree zero, moreover $i_{n}^{m}\left(\operatorname{Div}\left(F_{m}\right)\right)$ is $\Gamma_{m}^{n}$-invariant.

The following proposition gives us information on injectivity and surjectivity of $N$ and $i$.

Proposition 5.18. Let $F_{0} \subseteq K \subset E \subset \mathcal{F}$ with $\left[E: F_{0}\right]$ finite. Then

(a) the norm map $N_{K}^{E}: \mathcal{C} \ell^{0}(E) \rightarrow \mathcal{C} \ell^{0}(K)$ is surjective;

(b) the map $i_{E}^{K}: \mathcal{C} \ell^{0}(K) \rightarrow \mathcal{C} \ell^{0}(E)$ is injective.

Proof. We recall that $d_{\infty}=1$ and that $\infty$ is totally split in $\mathcal{F} / F_{0}$, hence all primes in $E$ and $K$ dividing $\infty$ have degree 1 as well.

(a) This is just an application of class field theory for function fields, see e.g. [2, Lemma 5.4 part (3)].

(b) Let $G:=\operatorname{Gal}(E / K)$ and, for any field $L$, let $\mathcal{P}_{L}$ be the principal divisors of $L$. Taking the $G$-cohomology in the exact sequence

$$
0 \longrightarrow \mathbb{F}_{q}^{\times} \longrightarrow E^{\times} \longrightarrow \mathcal{P}_{E} \longrightarrow 0
$$

we get

$$
0 \longrightarrow \mathbb{F}_{q}^{\times} \longrightarrow K^{\times} \longrightarrow \mathcal{P}_{E}^{G} \longrightarrow 0
$$

and $H^{1}\left(G, \mathcal{P}_{E}\right)=0$ (because of Hilbert 90 and $\left(|G|, \mathbb{F}_{q}^{\times}\right)=1$ ). Comparing this with the analogue of (17) for $K$ we have that $\mathcal{P}_{E}^{G}=\mathcal{P}_{K}$. Taking the $G$-cohomology in

$$
0 \longrightarrow \mathcal{P}_{E} \longrightarrow \operatorname{Div}^{0}(E) \longrightarrow \mathcal{C} \ell^{0}(E) \longrightarrow 0,
$$

we obtain

$$
0 \longrightarrow \mathcal{P}_{E}^{G}=\mathcal{P}_{K} \longrightarrow \operatorname{Div}^{0}(E)^{G} \longrightarrow \mathcal{C} \ell^{0}(E)^{G} \longrightarrow H^{1}\left(G, \mathcal{P}_{E}\right),
$$


which fits into the following commutative diagram

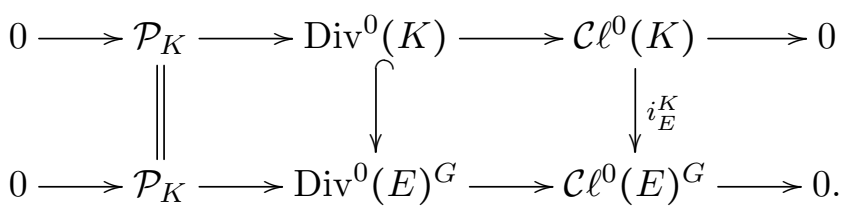

Applying the snake lemma we obtain the thesis.

From diagram (18) we also deduce that

$$
\mathcal{C} \ell^{0}(E)^{G} / i_{E}^{K}\left(\mathcal{C} \ell^{0}(K)\right) \simeq \operatorname{Div}^{0}(E)^{G} / i_{E}^{K}\left(\operatorname{Div}^{0}(K)\right) .
$$

To perform a limit and move to the infinite level we still have to deal with the kernel of the norm map $N_{K}^{E}(\chi): \mathcal{C} \ell^{0}(E)(\chi) \rightarrow \mathcal{C} \ell^{0}(K)(\chi)$ for characters of type 1 or 2 .

Lemma 5.19. Let $F_{0} \subseteq K \subset E \subset \mathcal{F}$ with $\left[E: F_{0}\right]$ finite and let $G:=\operatorname{Gal}(E / K)$. Assume $|G|=p$, then the group $\Delta:=\operatorname{Gal}\left(F_{0} / H_{A}\right)$ acts trivially on $\mathcal{C} \ell^{0}(E)^{G} / i_{E}^{K}\left(\mathcal{C} \ell^{0}(K)\right)$.

Proof. The composition of two natural maps

$$
\operatorname{Div}^{0}(E)^{G} \rightarrow \operatorname{Div}(E)^{G} \rightarrow \operatorname{Div}(E)^{G} / i_{E}^{K}(\operatorname{Div}(K)),
$$

has kernel $\operatorname{Div}^{0}(E)^{G} \cap i_{E}^{K}(\operatorname{Div}(K))=i_{E}^{K}\left(\operatorname{Div}^{0}(K)\right)$ and induces an injection

$$
\operatorname{Div}^{0}(E)^{G} / i_{E}^{K}\left(\operatorname{Div}^{0}(K)\right) \hookrightarrow \operatorname{Div}(E)^{G} / i_{E}^{K}(\operatorname{Div}(K)) .
$$

Thus it is enough to show that $\Delta$ acts trivially on $\operatorname{Div}(E)^{G} / i_{E}^{K}(\operatorname{Div}(K))$. Let $\mathfrak{p}_{1}, \ldots, \mathfrak{p}_{s}$ (resp. $\left.\mathfrak{P}_{1}, \ldots, \mathfrak{P}_{s}\right)$ be the set of primes of $K$ (resp. $E$ ) lying above $\mathfrak{p}$. The extension $E / K$ is totally ramified at $\mathfrak{p}$ and we can assume that $\mathfrak{P}_{j}$ is the unique prime of $E$ lying above $\mathfrak{p}_{j}$, i.e. $i_{E}^{K}\left(\mathfrak{p}_{j}\right)=p \mathfrak{P}_{j}$. Moreover the only extension where the prime $\mathfrak{p}$ may split is $H_{A} / F$, so $s$ divides $h^{0}(F)$, i.e. is coprime with $p$. We can write $\operatorname{Div}(K)=\bigoplus_{\nu} \mathbb{Z} \nu$ (where $\nu$ runs through all the primes of $K)$ and $\operatorname{Div}(E)=\oplus_{\nu} H_{\nu}$, with $H_{\nu}=\bigoplus_{w \mid \nu} \mathbb{Z} w$. Now for the ramified primes we have $H_{\mathfrak{p}_{\mathfrak{j}}}=\mathbb{Z} \mathfrak{P}_{j}=H_{\mathfrak{p}_{j}}^{G}$ while, for the unramified ones, if we let $G_{\nu}$ be the decomposition group of $\nu$ in $G$, we have $H_{\nu}=\mathbb{Z}\left[G / G_{\nu}\right] w$, so that $H_{\nu}^{G}=i_{E}^{K}(\mathbb{Z} \nu)$. Therefore

$$
\begin{gathered}
\operatorname{Div}(E)^{G}=\bigoplus_{j=1}^{s} \mathbb{Z} \mathfrak{P}_{j} \oplus \bigoplus_{\nu \nmid \mathfrak{p}} i_{E}^{K}(\mathbb{Z} \nu), \\
i_{E}^{K}(\operatorname{Div}(K))=\bigoplus_{j=1}^{s} p \mathbb{Z P}_{j} \oplus \bigoplus_{\nu \nmid \mathfrak{p}} i_{E}^{K}(\mathbb{Z} \nu)
\end{gathered}
$$

and finally

$$
\operatorname{Div}(E)^{G} / i_{E}^{K}(\operatorname{Div}(K))=\bigoplus_{j=1}^{s}(\mathbb{Z} / p) \mathfrak{P}_{j} .
$$

Note that, for any set of integers $\alpha_{1}, \ldots, \alpha_{s}$ coprime with $p$, the classes $\alpha_{j} \mathfrak{P}_{j}$ with $j=$ $1, \ldots, s$ still generate $\operatorname{Div}(E)^{G} / i_{E}^{K}(\operatorname{Div}(K))$.

Now consider the subfield $E^{\Delta}$ of $E$ (resp. $K^{\Delta}$ of $K$ ) fixed by $\Delta$. Since $|\Delta|$ is prime with $p$, there is a canonical isomorphism $G^{\Delta}:=\operatorname{Gal}\left(E^{\Delta} / K^{\Delta}\right) \simeq G$ and, since $F_{0} / H_{A}$ is totally ramified at $\mathfrak{p}$, we still have exactly $s$ primes in $E^{\Delta}\left(\right.$ resp. $\left.K^{\Delta}\right)$ above $\mathfrak{p}$ : let $\mathfrak{P}_{j}^{\Delta}\left(\right.$ resp. $\left.\mathfrak{p}_{j}^{\Delta}\right)$ 
be those primes and, as above, assume $i_{E^{\Delta}}^{K^{\Delta}}\left(\mathfrak{p}_{j}^{\Delta}\right)=p \mathfrak{P}_{j}^{\Delta}$. With the same argument, we can prove

$$
\operatorname{Div}\left(E^{\Delta}\right)^{G^{\Delta}} / i_{E^{\Delta}}^{K^{\Delta}}\left(\operatorname{Div}\left(K^{\Delta}\right)\right)=\bigoplus_{j=1}^{s}(\mathbb{Z} / p) \mathfrak{P}_{j}^{\Delta} .
$$

To conclude note that $i_{E}^{E^{\Delta}}\left(\mathfrak{P}_{j}^{\Delta}\right)=|\Delta| \mathfrak{P}_{j}$; since $|\Delta|$ is coprime with $p$, these classes generate $\operatorname{Div}(E)^{G} / i_{E}^{K}(\operatorname{Div}(K))$ and clearly the action of $\Delta$ on them is trivial.

We use the previous lemma to prove

Proposition 5.20. Let $F_{0} \subseteq K \subset E \subset \mathcal{F}$ with $\left[E: F_{0}\right]$ finite and let $G:=\operatorname{Gal}(E / K)$. If $\chi \in \widehat{G_{0}}$ is of type 1 or 2 , then

$$
\operatorname{Ker}\left(N_{K}^{E}(\chi)\right)=I_{G} \mathcal{C} \ell^{0}(E)(\chi),
$$

where $I_{G}$ denotes the augumentation of $G$.

Proof. We proceed by induction on $|G|$, starting with the case $|G|=p$ (for $|G|=1$ there is nothing to prove). Since $\chi$ is not of type 3, it may be seen as a nontrivial character of $\Delta=\operatorname{Gal}\left(F_{0} / H_{A}\right)$ and, by the previous lemma,

$$
\left(\mathcal{C} \ell^{0}(E)^{G} / i_{E}^{K}\left(\mathcal{C} \ell^{0}(K)\right)\right)(\chi)=0, \quad \text { i.e. } \quad \mathcal{C} \ell^{0}(E)^{G}(\chi)=i_{E}^{K}\left(\mathcal{C} \ell^{0}(K)\right)(\chi) .
$$

Let $g$ be a generator of $G$, then $I_{G} \mathcal{C} \ell^{0}(E)(\chi)=(1-g) \mathcal{C} \ell^{0}(E)(\chi)$ and we also recall that the cyclicity of $G$ yields $I_{G} \mathcal{C} \ell^{0}(E)(\chi) \simeq \mathcal{C} \ell^{0}(E) / \mathcal{C} \ell^{0}(E)^{G}$. We have two exact sequences

$$
0 \longrightarrow \operatorname{Ker}\left(N_{K}^{E}(\chi)\right) \longrightarrow \mathcal{C} \ell^{0}(E)(\chi) \stackrel{N_{K}^{E}(\chi)}{\longrightarrow} \mathcal{C} \ell^{0}(K)(\chi) \longrightarrow 0,
$$

(exact by Proposition 5.18, part (a)), and

$$
0 \longrightarrow \mathcal{C} \ell^{0}(K)(\chi) \stackrel{i_{E}^{K}}{\longrightarrow} \mathcal{C} \ell^{0}(E)(\chi) \stackrel{1-g}{\longrightarrow} I_{G} \mathcal{C} \ell^{0}(E)(\chi) \longrightarrow 0
$$

(exact by what we noted above). Cardinalities yield $\left|\operatorname{Ker}\left(N_{K}^{E}(\chi)\right)\right|=\left|I_{G} \mathcal{C} \ell^{0}(E)(\chi)\right|$, and, since $I_{G} \mathcal{C} \ell^{0}(E)(\chi) \subseteq \operatorname{Ker}\left(N_{K}^{E}(\chi)\right)$, we have equality of the two groups.

For the inductive step assume $|G|=p^{l}>p$ and take an intermediate field $K \varsubsetneqq E^{\prime} \varsubsetneqq E$; put $G_{1}=\operatorname{Gal}\left(E / E^{\prime}\right)$ and $G_{2}=\operatorname{Gal}\left(E^{\prime} / K\right)$, so that $G_{1}$ and $G_{2}$ have cardinality strictly smaller than $p^{l}$. The inductive hypothesis yields

$$
\operatorname{Ker}\left(N_{E^{\prime}}^{E}(\chi)\right)=I_{G_{1}} \mathcal{C} \ell^{0}(E)(\chi)
$$

and

$$
\operatorname{Ker}\left(N_{K}^{E^{\prime}}(\chi)\right)=I_{G_{2}} \mathcal{C} \ell^{0}\left(E^{\prime}\right)(\chi) .
$$

By Proposition 5.18 part (a), the norm $N_{E^{\prime}}^{E}: \mathcal{C} \ell^{0}(E)(\chi) \rightarrow \mathcal{C} \ell^{0}\left(E^{\prime}\right)(\chi)$ is surjective and so

$$
N_{E^{\prime}}^{E}\left(I_{G} \mathcal{C} \ell^{0}(E)(\chi)\right)=I_{G_{2}} \mathcal{C} \ell^{0}\left(E^{\prime}\right)(\chi) \text {. }
$$

Let $x \in \operatorname{Ker}\left(N_{K}^{E}(\chi)\right)$, since $N_{K}^{E}=N_{K}^{E^{\prime}} \circ N_{E^{\prime}}^{E}$, we have that $N_{E^{\prime}}^{E}(x) \in \operatorname{Ker}\left(N_{K}^{E^{\prime}}(\chi)\right)=$ $I_{G_{2}} \mathcal{C} \ell^{0}\left(E^{\prime}\right)(\chi)$, so there exists $\alpha \in I_{G}$ and $y \in \mathcal{C} \ell^{0}(E)(\chi)$ such that $N_{E^{\prime}}^{E}(x)=N_{E^{\prime}}^{E}(\alpha y)$. Therefore $x-\alpha y \in \operatorname{Ker}\left(N_{E^{\prime}}^{E}(\chi)\right)$, which yields

$$
x \in \operatorname{Ker}\left(N_{E^{\prime}}^{E}(\chi)\right)+I_{G} \mathcal{C} \ell^{0}(E)(\chi)=I_{G} \mathcal{C} \ell^{0}(E)(\chi),
$$


since $\operatorname{Ker}\left(N_{E^{\prime}}^{E}(\chi)\right)=I_{G_{1}} \mathcal{C} \ell^{0}(E)(\chi) \subseteq I_{G} \mathcal{C} \ell^{0}(E)(\chi)$. We have proved that

$$
\operatorname{Ker}\left(N_{K}^{E}(\chi)\right) \subseteq I_{G} \mathcal{C} \ell^{0}(E)(\chi)
$$

and the other inclusion is trivial.

We can finally prove the main theorems on $C_{\infty}$.

Theorem 5.21. Let $\chi \in \widehat{G_{0}}$ be a character of type 1 or 2 . Then $C_{\infty}(\chi)$ is a finitely generated $\Lambda:=W \llbracket \Gamma_{\infty} \rrbracket$-module. Moreover, let

$$
\Theta_{\infty}^{\sharp}(1, \chi)= \begin{cases}\Theta_{\infty}(1, \chi) & \text { if } \chi \text { is of type } 1, \\ \left(\frac{\Theta_{\infty}\left(\gamma^{-1}, \chi\right)}{1-\gamma^{-1}}\right)_{\left.\right|_{\gamma=1}} & \text { if } \chi \text { is of type } 2,\end{cases}
$$

be the inverse limit of the elements $\Theta_{n}^{\sharp}(1, \chi)$ appearing in Theorem 5.1\%; if $\Theta_{\infty}^{\sharp}(1, \chi) \neq 0$, then $C_{\infty}(\chi)$ is $\Lambda$-torsion.

Proof. By Proposition 5.18 part (a) and Proposition 5.20 we have

$$
C_{m}(\chi)=N_{m}^{n}\left(C_{n}(\chi)\right) \simeq C_{n}(\chi) / \operatorname{Ker}\left(N_{m}^{n}\right)=C_{n}(\chi) / I_{\Gamma_{m}^{n}} C_{n}(\chi) .
$$

Recall that $\mathfrak{I}_{m}=\lim _{\leftarrow} I_{\Gamma_{m}^{n}}$ is the augumentation ideal of $\operatorname{Gal}\left(\mathcal{F} / F_{m}\right)$ in $\Lambda$. The previous equality holds for every $n>m$, so

$$
C_{m}(\chi) \simeq C_{\infty}(\chi) / \Im_{m} C_{\infty}(\chi) .
$$

The module on the left is a finitely generated torsion $\Lambda / \mathfrak{I}_{m}=W\left[\Gamma_{m}\right]$-module. By the generalized Nakayama Lemma of [3] we obtain that $C_{\infty}(\chi)$ is a finitely generated $\Lambda$ module.

For the second part just note that $\Theta_{n}^{\sharp}(1, \chi) C_{n}(\chi)=0$ for every $n$, hence $\Theta_{\infty}^{\sharp}(1, \chi) C_{\infty}(\chi)=$ 0 , and $C_{\infty}(\chi)$ is a torsion $\Lambda$-module.

We conclude with the Main Conjecture.

Theorem 5.22 (Main Conjecture). Let $\chi \in \widehat{G_{0}}$ be a character of type 1 or 2 . Then

$$
\operatorname{Fitt}_{\Lambda}\left(C_{\infty}(\chi)\right)=\left(\Theta_{\infty}^{\sharp}(1, \chi)\right) .
$$

Proof. The proof follows the path of the one of Theorem 5.14. The equality

$$
\left(\Theta_{\infty}^{\sharp}(1, \chi)\right)=\lim _{\leftarrow}\left(\Theta_{n}^{\sharp}(1, \chi)\right)=\lim _{\leftarrow} \operatorname{Fitt}_{W\left[\Gamma_{n}\right]}\left(C_{n}(\chi)\right)
$$

reduces the statement to

$$
\operatorname{Fitt}_{\Lambda}\left(C_{\infty}(\chi)\right)=\lim _{\leftarrow} \operatorname{Fitt}_{W\left[\Gamma_{n}\right]}\left(C_{n}(\chi)\right) .
$$

Recall $C_{m}(\chi)=C_{\infty}(\chi) / \mathfrak{I}_{m} C_{\infty}(\chi)$, let $t_{1}, \ldots, t_{r}$ be $\Lambda$-generators of $C_{\infty}(\chi)$ and denote by $N_{m}^{\infty}: C_{\infty}(\chi) \rightarrow C_{m}(\chi)$ the projection so that $N_{m}^{\infty}\left(t_{1}\right), \ldots, N_{m}^{\infty}\left(t_{r}\right)$ generate $C_{m}(\chi)$. For any $n$ we have an exact sequence

$$
0 \longrightarrow K_{n} \longrightarrow W\left[\Gamma_{n}\right]_{28}^{\oplus r} \longrightarrow C_{n}(\chi) \longrightarrow 0
$$


where the map on the right is given by $\left(w_{1}, \ldots, w_{r}\right) \mapsto \sum_{i} w_{i} N_{n}^{\infty}\left(t_{i}\right)$ and $K_{n}$ is its kernel. They fit into the diagram

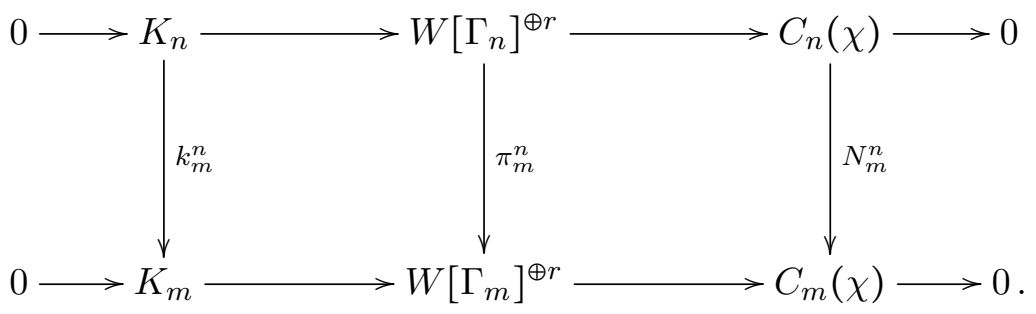

The kernel of $\pi_{m}^{n}$ is $\left(I_{\Gamma_{m}^{n}} W\left[\Gamma_{n}\right]\right)^{\oplus r}$ and, by Proposition 5.20, $\operatorname{Ker}\left(N_{m}^{n}\right)=I_{\Gamma_{m}^{n}} C_{n}(\chi)$, so the map between them is surjective. Moreover $\pi_{m}^{n}$ is surjective thus, by the snake lemma, $k_{m}^{n}$ is surjective as well and the diagram satisfies the Mittag-Leffler condition. Taking the inverse limit and comparing it with the analog of 20 for $K_{\infty}$ we get $K_{\infty}=\lim _{\leftarrow} K_{n}$.

To conclude the proof, one just follows the same technical arguments of the second part of the proof of Theorem 5.14 .

From this Main Conjecture and the interpolation formulas of the previous sections, one can derive a number of relations with special values of $L$ or Zeta-functions. For example we have seen in the proofs of Theorems 4.4 and 4.9 , that for non negative integers $i$ and $j$ with $i \equiv j\left(\bmod q^{d_{\nu}}-1\right)$

$$
\zeta_{A}\left(-s_{j}\right)\left(1-\mathfrak{p}^{s_{j}}\right)=Z(1, j)\left(1-\mathfrak{p}^{s_{j}}\right)=L_{\mathfrak{p}}\left(1, j, \omega^{i}\right)=\prod_{\nu \notin S}\left(1-\omega(\nu)^{i}\langle\nu\rangle_{\mathfrak{p}}^{j}\right)^{-1}=\Psi_{j, i}\left(\Theta_{S}(1)\right),
$$

where the extra factors at the ramified primes different from $\mathfrak{p}$ and $\infty$ disappear because in our case $S=\{\mathfrak{p}, \infty\}$. Taking $\chi$-parts and projecting from $G_{S}$ to $\Gamma_{\infty}$ via $\pi_{\infty}^{S}$, one gets

$$
\chi\left(\pi_{\infty}^{S}\left(L_{\mathfrak{p}}\left(X, j, \omega^{i}\right)(1)\right)=\Psi_{j, i}\left(\Theta_{\infty}(1, \chi)\right)\right.
$$

for characters of type 1. Up to now there are, to our knowledge, very few nonvanishing results on special values for function fields (see e.g. [1, Theorem E] and the discussion on "trivial zeroes" in [14, Section 8.13]): it would be interesting to see if these relations can shed some light on the subject for $L_{\mathfrak{p}}\left(1, j, \omega^{i}\right)$, which, as seen above, is basically equivalent to $C_{\infty}$ being a torsion $\Lambda$-module.

For $F=\mathbb{F}_{q}(t)$, in [2, Section 6] the authors present arithmetic information on some Bernoulli-Goss numbers, i.e. on special values of the Goss Zeta-function at integers: in our setting one should probably consider the finite $\mathbb{F}_{q}[t]$-module $H(\Phi / A)$ defined by Taelman in [27] (where $\Phi$ is a Drinfeld module over $A$ ), which plays the role of the ideal class group of a number field. For $F=\mathbb{F}_{q}(t)$ the Bernoulli-Goss numbers are linked to the isotypical components of $H(\Phi / A)$ by [28, Theorem 1 and Section 10]. It would be interesting to study inverse limits of $\mathfrak{p}$-parts of Taelman's modules associated to the fields $F_{n}$ as objects over the Iwasawa algebra: it is not clear whether this would lead to special values of our $\mathfrak{p}$-adic $L$-function or of some other (yet to be defined) $\mathfrak{p}$-adic analytic function (possibly another incarnation of the Stickelberger series).

Acknowledgements. We are grateful to Bruno Anglés, Francesc Bars and Ignazio Longhi for several useful conversations, suggestions and comments which provided invaluable contributions to the development of this paper. We would like to thank Fabrizio Andreatta 
and Marco Seveso for their help and support. We also thank the anonymous referees for their remarks which improved the exposition and provided inputs for future research.

\section{REFERENCES}

[1] B. Anglés - L. Taelman, "Arithmetic of characteristic $p$ special $L$-values" (with an appendix by V. Bosser) Proc. Lond. Math. Soc. (3) 110 (2015), 1000-1032.

[2] B. Anglés - A. Bandini - F. Bars - I. Longhi, "Iwasawa Main Conjecture for the Carlitz cyclotomic extension and applications", Math. Ann. 376, Issue 1-2 (2020), 475-523.

[3] P.N. Balister - S. Howson, "Note on Nakayama's lemma for compact $\Lambda$-modules", Asian J. Math. 1 (1997), no. 2, 224-229.

[4] A. Bandini - F. Bars - E. Coscelli, "Fitting ideals of class groups in Carlitz-Hayes cyclotomic extensions", to appear in J. Number Theory.

[5] A. Bandini - F. Bars - I. Longhi, "Characteristic ideals and Iwasawa theory", New York J. Math 20 (2014), 759-778.

[6] A. Bandini - F. Bars - I. Longhi, "Characteristic ideals and Selmer groups", J. Number Theory 157 (2015), 530-546.

[7] A. Bandini - M. Valentino, "Control theorems for $\ell$-adic Lie extensions of global function fields", Ann. Sc. Norm. Super. Pisa Cl. Sci. XIV (2015), no. 4, 1065-1092.

[8] A. Bandini - M. Valentino, "Euler characteristic and Akashi series for Selmer groups over global function fields", J. Number Theory 193 (2018), 213-234.

[9] D. Burns, "Congruences between derivatives of geometric L-functions." With an appendix by Burns, K.F. Lai and K.-S. Tan, Invent. Math. 184 (2011), no. 2, 221-256.

[10] L. Carlitz, "On certain functions connected with polynomials in a Galois field", Duke Math. J. (1935), 137-168.

[11] P. Cornacchia - C. Greither, "Fitting ideals of class group of real fields with prime power conductor", J. Number Theory 73 (1998), 459-471.

[12] E. Coscelli, "Stickelberger series and Iwasawa Main Conjecture for function fields", PhD Th., University of Milan (2018), avaliable at https://air.unimi.it/handle/2434/561439\#.XUWhDHvONPY .

[13] R. Crew, "L-functions of $p$-adic characters and geometric Iwasawa theory", Invent. Math. 88 (1987), no. 2, 395-403.

[14] D. Goss, Basic Structures of Function Field Arithmetic, (Springer-Verlag, 1996).

[15] D. Goss, " $v$-adic Zeta-functions, $L$-series and Measures for Function Fields", Invent. Math. 55 (1979), 107-116.

[16] C. Greither - M. Kurihara, "Stickelberger elements, Fitting ideals of class groups of CM-fields and dualisation", Math. Z. 260 (2008), no. 4, 905-930.

[17] C. Greither - C.D. Popescu, "The Galois module structure of $\ell$-adic realizations of Picard 1-motives and applications", Int. Math. Res. Not. (2012), no. 5, 986-1036.

[18] C. Greither - C.D. Popescu, "Fitting ideals of $\ell$-adic realizations of Picard 1-motives and class groups of global function fields", J. Reine Angew. Math. 675 (2013), 223-247.

[19] D.R. Hayes, "A brief introduction do Drinfeld Modules" in "The Arithmetic of function fields" (Columbus, OH, 1991) Ohio State Univ. Math. Res. Inst. Publ. 2, 1-32.

[20] K. Kato, "Iwasawa theory and generalizations", ICM Vol I, Eur. Math. Soc. Zürich, Vol. 12, 335-357 (2007).

[21] K.-F. Lai - I. Longhi - K.-S. Tan - F. Trihan, "The Iwasawa main conjecture for constant ordinary abelian varieties over function fields", Proc. Lond. Math. Soc. 112 (2016), , no. 6, 1040-1058.

[22] B. Mazur - A. Wiles, "Class fields of abelian extensions of $\mathbb{Q} "$, Invent. Math. 76 (1984), 179-330.

[23] D. Mumford, Abelian Varieties, Tata Inst. of Fundamental Research, Bombay, 1970.

[24] D.G. Northcott, Finite free resolutions, Cambridge University Press, Cambridge Tracts in Mathematics, No. 71, Cambridge, 1976.

[25] M. Rosen, Number theory in function fields, GTM 210, Springer-Verlag, New York, 2002.

[26] L. Shu, "Kummer's criterion over global function fields", J. Number Theory 49 (1994), 319-359.

[27] L. Taelman, "Special L-values of Drinfeld modules", Annals of Math. 175 (2012), 369-391.

[28] L. Taelman, "A Herbrand-Ribet theorem for function fields", Invent. Math. 188 (2012), 253-275. 
[29] K.-S. Tan, "A generalized Mazur's theorem and its applications", Trans. Amer. Math. Soc. 362 (2010), 4433-4450.

[30] K.-S. Tan, "Selmer groups over $\mathbb{Z}_{p}^{d}$-extensions", Math. Ann. 359 (2014), 1025-1075.

[31] J. Tate, Les conjectures de Stark sur les Fonctiones L d'Artin en $s=0$, Progress in Mathematics 47, Birkhäuser, (1984).

[32] D.S. Thakur, Function Field Arithmetic, World Scientific Publishing Co., Inc., River Edge, NJ, 2004.

Andrea Bandini: Università degli Studi di Pisa, Dipartimento di Matematica, Largo Bruno Pontecorvo, 5, 56127 PisA - Italy

Email address: andrea.bandini@unipi.it

EdoARdo Coscelli:

Email address: coscelliedoardo@gmail.com 